\title{
Boosters and Barriers for Direct Cardiac Reprogramming
}

${\text { Mahmood Talkhabi }{ }^{1 *} \text {, Elmira Rezaei Zonooz }{ }^{2} \text {, Hossein Baharvand }}^{2,3^{*}}$

1. Department of Animal Sciences and Biotechnology, Faculty of Life Sciences and Biotechnology, Shahid Beheshti University, Tehran, Iran

2. Department of Stem Cells and Developmental Biology, Cell Science Research Center, Royan Institute for Stem Cell Biology and Technology, ACECR, Tehran, Iran

3. Department of Developmental Biology, University of Science and Culture, Tehran, Iran *Corresponding authors:

Mahmood Talkhabi, Ph.D.

Department of Animal Sciences and Biotechnology, Faculty of Life Sciences and Biotechnology, Shahid Beheshti University, Tehran, Iran.

Tel: +98 2129902720

Fax: +98 2122431664

Email: m_talkhabi@sbu.ac.ir

Or

Hossein Baharvand, Ph.D.

Department of Stem Cells and Developmental Biology, Cell Science Research Center, Royan Institute for Stem Cell Biology and Technology, ACECR, P.O. Box 19395-4644, Tehran, Iran.

Tel: +98 2122306485

Fax: +98 2123562507

Email: Baharvand@RoyanInstitute.org

(C) 2017. This manuscript version is made available under the Elsevier user license http://www.elsevier.com/open-access/userlicense/1.0/ 


\begin{abstract}
Heart disease is currently the most significant cause of morbidity and mortality worldwide, which accounts for approximately $33 \%$ of all deaths. Recently, a promising and alchemy-like strategy has been developed called direct cardiac reprogramming, which directly converts somatic cells such as fibroblasts to cardiac lineage cells such as cardiomyocytes (CMs), termed induced CMs or iCMs. The first in vitro cardiac reprogramming study, mediated by cardiac transcription factors (TFs) -Gata4, Tbx5 and Mef2C-, was not enough efficient to produce an adequate number of fully reprogrammed, functional iCMs. As a result, numerous combinations of cardiac TFs exist for direct cardiac reprogramming of mouse and human fibroblasts. However, the efficiency of direct cardiac reprogramming remains low. Recently, a number of cellular and molecular mechanisms have been identified to increase the efficiency of direct cardiac reprogramming and the quality of iCMs. For example, microgrooved substrate, cardiogenic growth factors [VEGF, FGF, BMP4 and Activin A], and an appropriate stoichiometry of TFs boost the direct cardiac reprogramming. On the other hand, serum, TGF $\beta$ signaling, activators of epithelial to mesenchymal transition, and some epigenetic factors (Bmi1 and Ezh2) are barriers for direct cardiac reprogramming. Manipulating these mechanisms by the application of boosters and removing barriers can increase the efficiency of direct cardiac reprogramming and possibly make iCMs reliable for cell-based therapy or other potential applications. In this review, we summarize the latest trends in cardiac TF- or miRNA-based direct cardiac reprogramming and comprehensively discuses all molecular and cellular boosters and barriers affecting direct cardiac reprogramming.
\end{abstract}


Keywords: Direct cardiac reprogramming, Reprogramming boosters, Reprogramming barriers, Transcription factors, Induced cardiomyocytes, Signaling pathways, Epigenetic regulators 


\section{Introduction}

The adult human heart does not regenerate significant amounts of lost cardiomyocytes (CMs) after injury or diseases such as myocardial infarction (MI) [1]. Given the poor cardiac regenerative capacity of adult mammals, it is understandable that heart disease is the most significant cause of morbidity and mortality worldwide, which accounts for approximately $33 \%$ of all deaths [2]. Cardiac cell-based therapy is a promising and achievable strategy to treat cardiac diseases because of accessibility to a variety of cell sources, the ability to scale-up desired cells in vitro, and preliminary promising results obtained from cell transplantation in animal models [3]. To this end various cell populations such as bone marrow-hematopoietic stem cells, mesenchymal stem cells (MSCs), pluripotent stem cell (PSC)-derived CM, PSC-derived cardiac progenitor cells (CPCs), and adult cardiac stem cells are potential cell sources for replacement therapy [4]. Surprisingly, within a year after the first study of bone marrow cells in an experimental MI in 2001, these cells were used for patients. It took only 3 years from the first use of stem cells (skeletal myoblasts) in an animal model of Heart Failure to the initial use of these cells in humans [5]. Few ideas in medicine have been translated from the laboratory to the clinic faster than the application of stem cells to treat cardiac diseases. However, the emerging results from stem cell therapy are highly controversial and somehow disappointing.

During the past 6 years, a new strategy has been highlighted for production of de novo cardiac lineage cells (CPCs/CMs) required in cardiac cell-based therapy. This alchemy like strategy is called direct cardiac reprogramming, in which non-cardiac somatic cells are directly converted to $\mathrm{CPCs} / \mathrm{CMs}$, but without entering pluripotent state $[5,6]$. Therefore, this strategy not only removes human PSC associated tumorigenicity risk, but also solves the immunogenicity problem that is a big concern in cell-based therapy. Since patient-derived somatic cells (e.g., skin-derived 
fibroblasts) are the starting cells in this strategy, it resolves the ethical issues connected to embryonic stem cells (ESCs). However, for clinical applications of direct cardiac reprogramming, some issues must be resolved. Currently the efficiency of iCM production via direct reprogramming is low (discussed later) and the produced iCMs are not enough mature for use in cardiac cell-based therapy. More importantly, most of the methods are being used for producing iCMs are retro- and lentivirus based, which decrease the safety of direct cardiac reprogramming strategy. It seems necessary to develop strategies that increase safety, maturity, and quantity of iCMs. Direct cardiac reprogramming strategies mediated by growth factors, small molecules or protein transduction will be more safe than retro- or lentiviruses based strategies. Moreover, it seems that stepwise direct cardiac reprogramming strategies produce more mature iCMs required for potential applications. In his review, we summarize in detail all direct cardiac reprogramming studies that have relied on the overexpression of cardiac specific factors [transcription factors (TFs) and/or miRNAs]. We comprehensively focus on cellular and molecular mechanisms that boost and obstruct the cardiac reprogramming. We hope that this upto-date review will shed light on mechanisms of direct cardiac reprogramming, as well as conduct new studies for efficiently reprogramming somatic cells into cardiac cells and even other lineage cells. 
Table 1. List of abbreviations and descriptions used throughout the paper

\begin{tabular}{|c|c|c|c|}
\hline Abbreviation & Description & Abbreviation & Description \\
\hline 7F2i & $\begin{array}{l}\text { Gata4, Mef2C, Tbx5, Esrrg, Mesp1, Myocd, Zfpm2, } \\
\text { SB431542 and XAV }\end{array}$ & hiCPCs & Human induced cardiac progenitor cells \\
\hline ACFs & Adult cardiac fibroblasts & HMT & Histone methyltransferase complex \\
\hline $\mathrm{AcH} 3$ & Histone 3 acetylation & iCMs & Induced cardiomyocytes \\
\hline ADFs & Adult dermal fibroblasts & iPSCs & Induced pluripotent stem cells \\
\hline ADMSCs & Adipose-derived mesenchymal stem cells & Jasp & Jasplakinolide \\
\hline Bleb & Blebbistatin & LIF & Leukemia inhibitory factor \\
\hline BMP4 & Bone morphogenetic protein 4 & LSGM & Low serum growth medium \\
\hline CGM & Cardiomyocyte growth medium & M3 & M3 transactivation domain of MyoD \\
\hline CM & Cardiomyocyte & MEFs & Mouse embryonic fibroblasts \\
\hline CMs & Cardiomyocytes & $\mathrm{Ml}$ & Myocardial infarction \\
\hline CytoD & Cytochalasin D & miRNA & MicroRNA \\
\hline DCR & Direct cardiac reprogramming & MM3 & Mef2C fused to M3 (Mef2C-M3) \\
\hline Dkk1 & Dickkopf1 & MM3HGT & Mef2C fused to M3, Hand2, Gata4 and Tbx5 \\
\hline EMT & Epithelial-to-mesenchymal transition & MSCs & Mesenchymal stem cells \\
\hline ESCs & Embryonic stem cells & p38 MAPK & P38 mitogen-activated protein kinase \\
\hline FGF & Fibroblast growth factor & piCPCs & Protein-induced cardiac progenitor cells \\
\hline FGM & Fibroblast growth medium & PRC2 & Polycomb Repressive Complex 2 \\
\hline GMT & Mef2C, Gata4 and Tbx5 & PSC & Pluripotent stem cells \\
\hline GMT2i & Mef2C, Gata4 and Tbx5, SB431542 and XAV & PSC-CMs & Pluripotent stem cell-derived cardiomyocytes \\
\hline GMTM2i/4F2i & Gata4, Mef2C, Tbx5, Myocd, SB431542 and XAV & $\mathrm{RM}$ & Reprogramming median \\
\hline GMTMM & Gata4, Mef2C, Tbx5, Mesp1 and Myocd & SB & SB431542 \\
\hline GMTNB & Gata4, Mef2C, Tbx5, Nkx2.5 and Baf60c & SP & Side population \\
\hline HCFs & Human cardiac fibroblasts & TFs & Transcription factors \\
\hline HDFs & Human dermal fibroblasts & TGF $\beta$ & Transforming growth factor beta \\
\hline HFFs & Human foreskin fibroblasts & TrxG & Trithorax Group proteins \\
\hline HGMT & Gata4, Hand2, Mef2C and Tbx5 & TTFs & Tail tip fibroblasts \\
\hline HGMT2m & Hand2, Gata4, Mef2C, Tbx5, miR-1 and miR-133 & VEGF & Vascular endothelial growth factor a \\
\hline HGMTN & Hand2, Gata4, Mef2C, Tbx5 and Nkx2.5 & \multirow[t]{2}{*}{ VPA } & \multirow[t]{2}{*}{ Valproic acid } \\
\hline hiCMs & Human induced cardiomyocytes & & \\
\hline
\end{tabular}

\section{Direct reprogramming}

During normal development and differentiation, stem cells (e.g., PSCs) gradually lose their potency, enter differentiation paths, and form stem cells with reduced potency or fully differentiated cells (Figure 1). During cardiac development, the fate of cardiac lineage cells is established and maintained by complex regulatory networks of extrinsic (signaling molecules) and intrinsic (TFs and miRNAs) factors that promote expression of cell type-specific gene products and repress regulators of other lineages [7]. Once established, cellular identity is remarkably stable despite numerous intrinsic and extrinsic perturbations. This cell stability is the result of combined multiple molecular factors that include cis-acting epigenetic modifications such as DNA methylation, post-translational modifications of histone tails, nucleosome 
positioning, incorporation of histone variants into nucleosomes, and trans-acting regulatory factors such as DNA-binding TFs, transcriptional coactivators, noncoding RNAs, and chromatin remodeling complexes [7, 8]. Once scientists understood the mechanisms that control cell fates, they have been encouraged to attempt to establish new cell fates in different cell types by forced expressions of TFs and miRNAs involved in the establishment and maintenance of a distinct cellular lineage. In 1988, researchers found that overexpression of MyoD, a myogenic TF, could convert fibroblasts into myoblasts [9]. The extraordinary reprogramming ability of $\mathrm{MyoD}$ demonstrated that this single TF had the capability to reprogram non-mesoderm cells such as neural and liver cells to a myogenic phenotype [10]. Meanwhile, studies in model organisms showed that the forced expression of individual master regulatory TF containing homeobox domains could induce the formation of complex body structures. For example, overexpression of eyeless, the fly ortholog of Pax6, induced ectopic eye formation on the legs of drosophila [11]. The concept of cell fate reprogramming that bypasses normal development appeared promising for regenerative medicine. In 2006, Yamanaka et al. studied cell fate reprogramming (Figure 1) where they reported that a fully differentiated somatic cell (e.g., fibroblast) could be reprogrammed to a PSC (known as iPSCs) by overexpression of only four TFs (c-Myc, Oct4, Sox2, and Klf4 or OSKM) [12, 13]. Yamanaka's discovery showed that considerable changes could occur in the very stable differentiated state. Abundant evidences have demonstrated that iPSCs can be efficiently differentiated into multiple cell types such as CMs which can be used for regenerative therapies, disease modeling, and drug discovery [2]. A key lesson from iPSC reprogramming is that a combination of regulators (TFs, miRNAs, and signaling pathways) involved in the establishment of a cell fate/identity could be sufficient to induce a new cell fate in another cell type. Therefore, many researchers began to change the fate of mature somatic 
cells to progenitor cells or other mature cells [14]. Today this type of cell conversion is known as transdifferentiation, direct reprogramming, direct conversion, and cell fate switching (Figure 1)[15]. This strategy bypasses the pluripotent stage and increases the safety of produced cells. Zhou et al. have first demonstrated this reprogramming strategy by directly converting exocrine pancreatic cells into insulin-producing endocrine cells in the mouse pancreas with three TFs (Ngn3, Pdx, and MafA) [16]. Successful direct reprogramming has been reported for many cell types, including pancreatic beta cells [17], neurons [18], hepatocyte-like cells [19], and hematopoietic progenitor cells [20]. Unlike direct reprogramming with multiple cell specific factors, numerous reports indicate that iPSC specific TFs can be used for direct reprogramming [21]. To avoid the emerging pluripotent cells during direct reprogramming, OSKM are overexpressed for a short time that is required for generating unstable intermediated cells, which can be programmed into the desired cell types [22, 23]. For example, combining Oct4 expression with administration of selected cytokines can reprogram human fibroblasts into hematopoietic progenitors [24], whereas transient expressions of OSKM followed by Jak/Stat inhibition results in the emergence of beating iCMs [25]. We intend to discuss in detail the advances in cardiac reprogramming mediated by cardiac specific factors (TFs and miRNAs), rather than iPSC factors. 


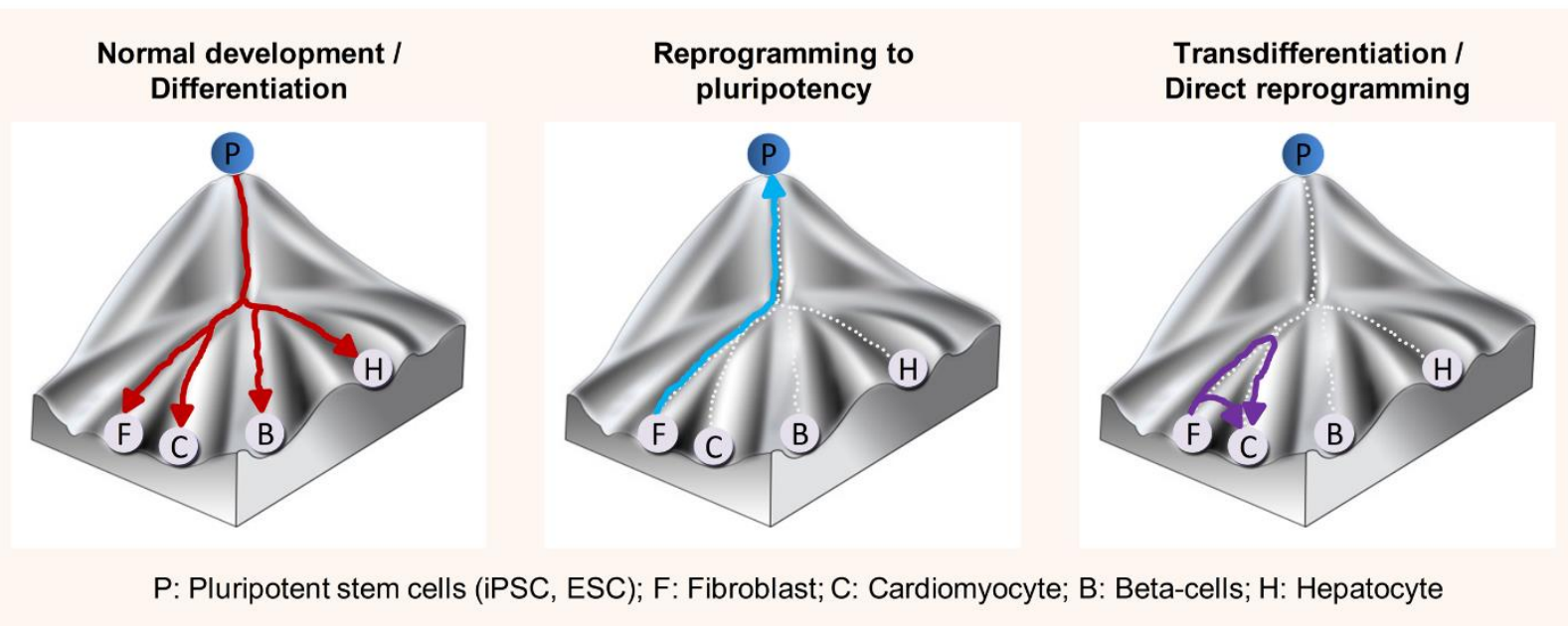

Figure 1. Generation of de novo cell types such as CMs on Waddington's epigenetic landscape. Normal development/differentiation of a cell. A PSC (P) rolls down, gradually loses its differentiation potential, and generates a new terminally differentiated cell (F, C, H, and B). Reprogramming to pluripotency. A fully differentiated cell (F) climbs the developmental incline to reach the non-differentiated state and regains pluripotency (P). Direct reprogramming, in which a fully differentiated cell (F) jumps a lower hill to become a new terminally differentiated cell (C). In addition, the differentiated cell might climb to a progenitor state, and then follows the developmental path to become the terminally differentiated cell (C).

\section{Direct cardiac reprogramming in vitro}

Before 2010, developmental biologists had determined a number of cardiac development properties as well as in vitro CM differentiation from PSCs. Researchers gained a thorough understanding of the cardiogenesis regulatory network that consisted of signaling pathways, TFs, miRNAs, and other factors, which control CM development, function, and maturation. Scientists have understood that in this network, factors often function in common complexes and the mutations that disrupt their interaction can lead to similar forms of heart malformations seen with mutations in Gata4 and Tbx5 [26, 27]. Researchers observed that overexpression of Gata4, Tbx5, and Baf60C (a chromatin remodeling protein) converted noncardiogenic mesoderm into beating 
CMs in vivo by a mechanism where Nkx2.5 was induced by Gata4 and Baf60C [28]. These findings along with the knowledge obtained from iPSC technology and direct reprogramming in other lineages encouraged Srivastava and colleagues searched for a combination of cardiac TFs that would reprogram mouse fibroblasts to CMs in vitro, which were called induced CMs (iCMs) [29]. They chose potential cardiac reprogramming factors by microarray analyses that identified TFs and epigenetic remodeling factors with greater expression in mouse CMs compared to cardiac fibroblasts at embryonic day 12.5 [30]. From these, 13 factors were chosen that exhibited severe developmental cardiac defects and embryonic lethality when mutated. They also included Mesp1, which had been shown to induce cardiac transdifferentiation in xenopus [31]. Srivastava and colleagues used cardiac fibroblasts (CFs) derived from a transgenic mouse ( $\alpha$ MHC-GFP) as starting cells and overexpressed 14 TFs and a pool of miRNAs in the cells [29]. Interestingly, their candidate miRNAs did not show any significant positive effects for direct cardiac reprogramming and were subsequently excluded from the study. Through serial deletions of one TF at a time, and quantification $\alpha \mathrm{MHC}-\mathrm{GFP}+$ cells, they found that GMT adequately induced GFP expression in $\sim 15 \%$ to $20 \%$ of the cells [29]. However, the majority of cells were partially reprogrammed, with $5 \%$ of the total infected cell population that expressed additional cardiac markers such as cardiac Troponin $\mathrm{T}$ (cTnT) and assembled sarcomere structures. Furthermore, only $\sim 0.5 \%$ of the $\alpha \mathrm{MHC}-\mathrm{GFP}+/ \mathrm{cTnT}+$ cells had the capability to beat (Table 2 ) [29]. They also reported that Gata4, Mef2C and Tbx5 (GMT) reprogrammed tail-tip fibroblasts (TTFs) into iCMs. They observed that GMT directly reprogrammed fibroblasts into iCMs without first becoming CPC expressing Is11 or Mesp1. The efficiency of generation of beating iCMs was only $0.01 \%-0.1 \%$ of the starting fibroblasts, which was similar to that seen for iPSC generation under original conditions [12]. After this initial report, Srivastava and colleagues and 
other researchers focused on some basic parts of this interesting field: 1) finding new combinations of TFs and/or miRNAs to increase the efficiency of direct cardiac reprogramming of mouse fibroblasts; 2) seeking new methods to analyze the efficiency of direct cardiac reprogramming; 3) direct cardiac reprogramming of mouse fibroblasts isolated from different sources; 4) direct cardiac reprogramming of human fibroblasts; and 5) in vivo cardiac reprogramming of CFs in animal models (Figure 2). These attempts resulted in many interesting and important findings. For example, Song et al. reported that GMT plus Hand2 (HGMT) enhanced cardiac reprogramming efficiency by threefold (Figure 2, Table 2) [32]. Similarly, Ifkovits et al. showed that addition of Nkx2.5 to HGMT and TGF $\beta$ inhibition increased the efficiency of direct cardiac reprogramming by approximately 50-fold compared to GMT alone (Figure 2, Table 2) [33]. Hirai et al. found that M3, the transactivation domain of MyoD, could be fused to Mef2C to form an active chimeric TF called MM3 (Table 2) [34]. MM3HGT efficiently increased cardiac reprogramming to 15-fold greater than HGMT without the MyoD's M3. On the other hand, Protze et al. [35] and Zhou et al. [36] identified that myocardin could be used instead of Gata4 to make a new combination which increased the reprogramming efficiency (Table 2). 
Table 2. Direct cardiac reprogramming in murine cells.

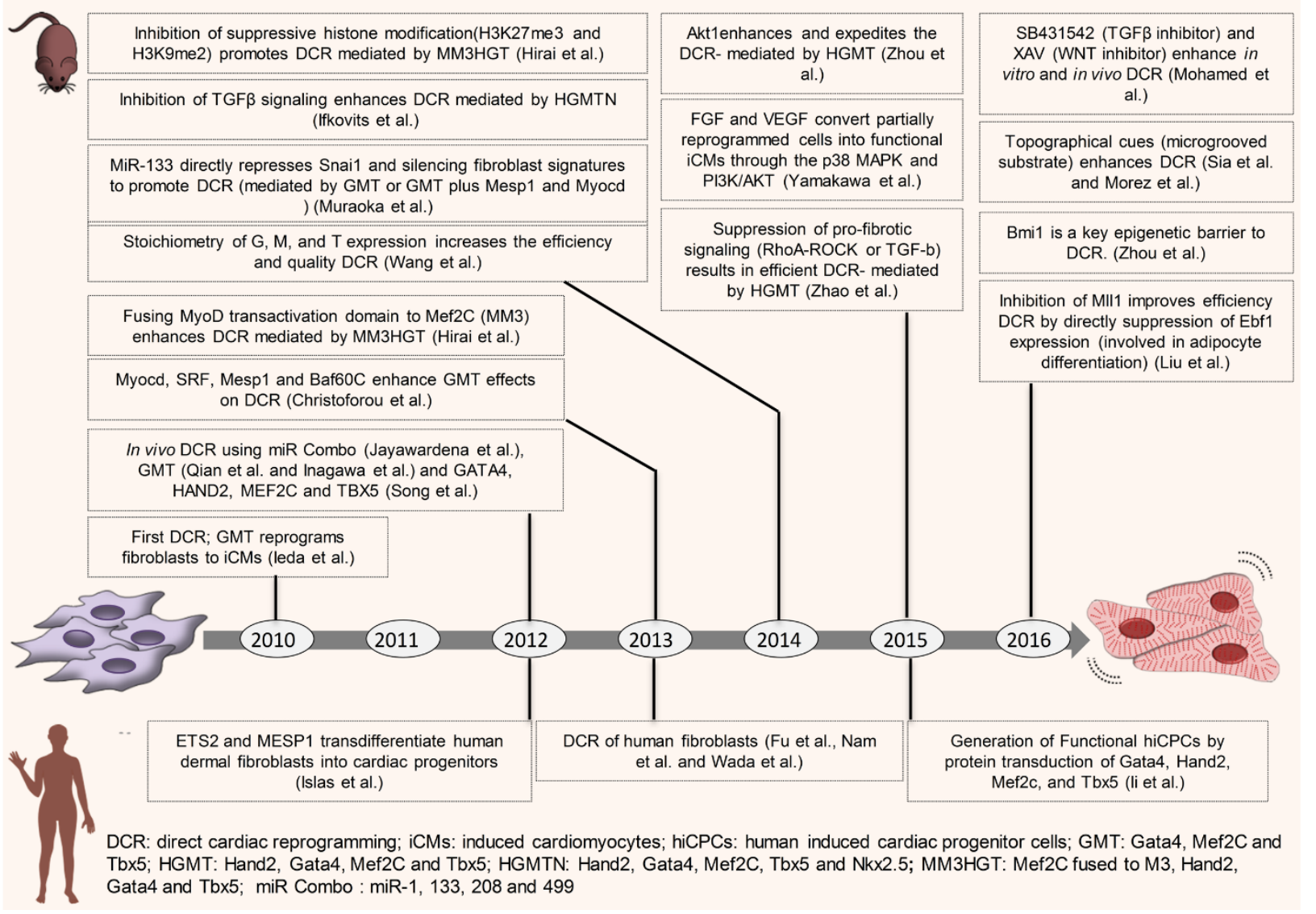

Figure 2. Timeline of direct cardiac reprogramming (since the initial report at 2010). 


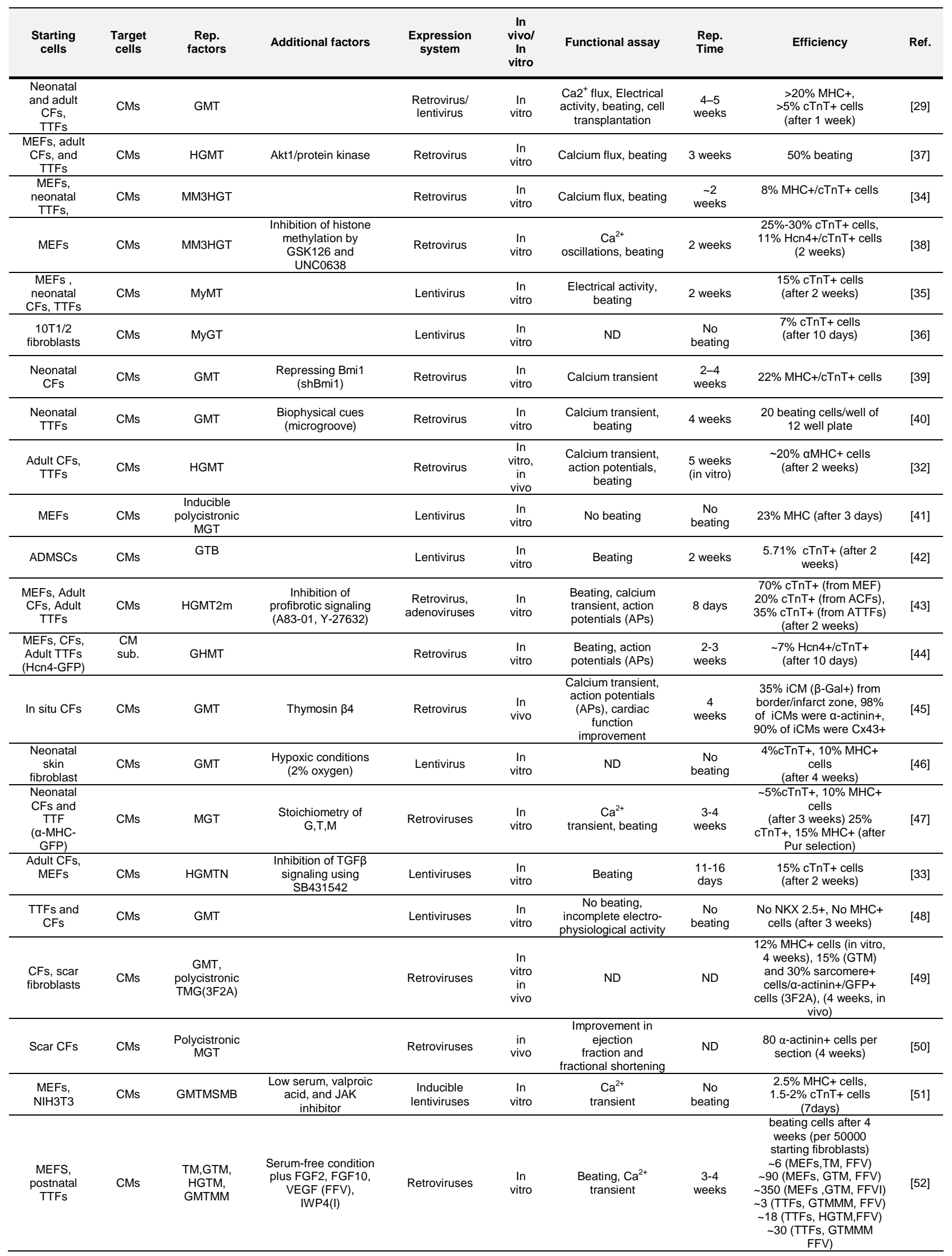




\begin{tabular}{|c|c|c|c|c|c|c|c|c|c|}
\hline $\begin{array}{l}\text { Neonatal } \\
\text { and adult } \\
\text { CFs, in situ } \\
\text { CFs }\end{array}$ & $\mathrm{CMs}$ & miR combo & JAK inhibitor I & $\begin{array}{c}\text { Transient } \\
\text { transfection of } \\
\text { mir combo (in } \\
\text { vitro) } \\
\text { Lentiviruses (in } \\
\text { vivo) } \\
\end{array}$ & $\begin{array}{l}\text { In } \\
\text { vitro } \\
\text { In } \\
\text { vivo }\end{array}$ & $\begin{array}{l}\text { Beating and } \mathrm{Ca}^{2+} \\
\text { transient (in vitro), } \\
\text { cardiac function } \\
\text { improvement (in } \\
\text { vivo) }\end{array}$ & $\begin{array}{c}3-4 \\
\text { weeks }\end{array}$ & $\begin{array}{c}28 \% \text { MHC+ cells (after } 7 \\
\text { days, in vitro) } \\
1 \% \text { iCM (after } 4 \text { weeks, } \\
\text { in vivo) }\end{array}$ & $\begin{array}{l}{[53,} \\
54]\end{array}$ \\
\hline MEFs & $\mathrm{CMs}$ & GMT & miR-133 & $\begin{array}{c}\text { Retroviruses } \\
\text { (GMT), } \\
\text { Synthetic } \\
\text { mimics mir133 } \\
\end{array}$ & $\begin{array}{l}\ln \\
\text { vitro }\end{array}$ & $\begin{array}{c}\text { Beating and } \mathrm{Ca}^{2+} \\
\text { transient }\end{array}$ & 10 days & $\begin{array}{c}25 \% \mathrm{MHC}+\text { cells, } 8 \% \\
\mathrm{MHC}+/ \mathrm{cTnT}+\text { cells (after } \\
1 \text { week) }\end{array}$ & [55] \\
\hline $\begin{array}{l}\text { Neonatal } \\
\text { CFs }\end{array}$ & CMs & GMT & & mRNA & $\begin{array}{l}\ln \\
\text { vitro }\end{array}$ & ND & ND & $\begin{array}{c}0.5 \% \mathrm{MHC}+\text { cells (after } 1 \\
\text { week) }\end{array}$ & [56] \\
\hline $\begin{array}{l}\text { Neonatal } \\
\text { TTFs and } \\
\text { CFs }\end{array}$ & $\mathrm{CMs}$ & $\mathrm{miR}$ combo & $\begin{array}{l}\text { Reprogramming } \\
\text { medium that } \\
\text { contained selenium }\end{array}$ & $\begin{array}{l}\text { Transient } \\
\text { transfection of } \\
\text { mir combo (in } \\
\text { vitro) } \\
\text { lentiviruses (In } \\
\text { vivo) }\end{array}$ & $\begin{array}{l}\text { In } \\
\text { vitro }\end{array}$ & ND & ND & $\begin{array}{c}\sim 23 \% \mathrm{MHC}+\text { cells, } \sim 13 \% \\
\text { cTnT+ cells (after } 2 \\
\text { weeks) }\end{array}$ & [57] \\
\hline $\begin{array}{l}\text { MEFs and } \\
\text { CFs }\end{array}$ & $\mathrm{CMs}$ & $\begin{array}{l}\text { Polycistronic } \\
\text { MGT }\end{array}$ & $\begin{array}{c}\text { MM408 } \\
\text { (MIl1 H3K4 methyl- } \\
\text { transferase) }\end{array}$ & $\begin{array}{l}\text { Polycistronic } \\
\text { retrovirus }\end{array}$ & $\begin{array}{l}\ln \\
\text { vitro }\end{array}$ & $\begin{array}{c}\text { Beating and } \mathrm{Ca}^{2+} \\
\text { transient }\end{array}$ & 3 weeks & $\begin{array}{c}>10 \% \text { cTnT+/aMHC+ } \\
\text { (day } 10), \\
\sim 45 \text { beating loci/1 } 1 \mathrm{~cm} 2 \text { (4 } \\
\text { weeks) }\end{array}$ & [58] \\
\hline
\end{tabular}

Rep. Factor: reprogramming factor; Rep. Time: Time required for beating initiation; ND: not determined; CM sub.; CM subtypes: atrial, ventricular, and pacemaker; CFs: cardiac fibroblasts: TTFs: tail tip fibroblasts; MEFs: mouse embryonic fibroblasts; ADMSCs: adipose-derived mesenchymal stem cells; GMT: GATA4, MEF2C, and TBX5; HGMT: Hand2, Gata4, Mef2C and Tbx5; MM3HGT: Mef2C fused to M3, Hand2 Gata4 and Tbx5; MyMT: Myocd, Mef2C and Tbx5; MyGT: Myocd, Gata4 and Tbx5; GTB: Gata4, Tbx5 and Baf60C; HGMT2m: Hand2, Gata4, Mef2C, Tbx5, miR-1 and miR-133; HGMTN: Hand2, Gata4, Mef2C, Tbx5 and Nkx2.5; GMTMSMB: Tbx5; GTB: Gata4, Tbx5 and Baf60C; HGMT2m: Hand2, Gata4, Mef2C, Tbx5, miR-1 and miR-133; HGMTN: Hand2, Gata4, Mef2C, Tbx5 and Nkx2.5; GMTMSMB:
Gata4, Mef2C, Tbx5, Myocd, SRF, Mesp1 and Baf60C; TM: Tbx5 and Mef2C; GMTMM: Gata4, Mef2C, Tbx5, Mesp1 and Myocd; miR combo: miRNA 1, 133, 208, and 499; SB: SB431542

Three years after the original report of mouse fibroblast reprogramming with GMT, researchers have published three papers where human fibroblasts could be directly reprogrammed to iCM in vitro (Figure 2, Table 3) [60-62]. Unlike iPSC TFs that act as reprogramming elements in mice, humans, rats, pigs, and chickens, it seems that the same direct reprogramming TFs cannot act in different species [63]. For example, neural reprogramming in human cells can be achieved using mouse reprogramming factors (Brn2, Ascl1, and Myt1l) in combination with other TF such as NeuroD1 and miRNAs (e.g., miR-9/9) [64]. Expectedly, it has been shown that GMT was inadequate for direct cardiac reprogramming in all types of human fibroblasts; new TFs or miRNAs were required to be added to the core of cardiac reprogramming (GMT). Ieda and colleagues showed that the addition of Mesp1 and Myocd to GMT (GMTMM) could change human cardiac fibroblasts (HCFs) and human dermal fibroblasts (HDFs) to iCMs that had rodlike or polygonal shapes (Table 3) [61]. The human iCMs (hiCMs) expressed multiple cardiacspecific proteins and sarcomeric structures, exhibited spontaneous $\mathrm{Ca}^{2+}$ oscillations, and matured 
to exhibit action potentials. These hiCMs could contract synchronously in coculture with murine CMs. Moreover, Srivastava and colleagues reported two new factors (ESRRG and MESP1) that could work with GMT to induce an epigenetically stable cardiac identity in human fibroblasts derived from different origins including human ESCs (hESC), human fetal heart and neonatal skin (Table 3) [60]. They showed that addition of myocardin and ZFPM2 to 5TFs (GMT, ESRRG and MESP1) enhanced reprogramming led to sarcomere formation, calcium transients, and action potentials. In the third report, Olson and colleagues showed that Hand2, Myocardin, miR-1, and miR-133 cooperate with Tbx5 and Gata4 to reprogram human fibroblasts [human foreskin fibroblasts (HFF)], adult cardiac fibroblasts (ACFs), and adult dermal fibroblasts (ADFs)] to hiCMs that displayed sarcomere-like structures and calcium transients, as well as spontaneous contraction (Table 3) [62]. Interestingly, they showed that Mef2C did not have any positive effect on cardiac reprogramming. Myocardin appeared to have a vital role in cardiac reprogramming in human fibroblasts, as it has been included in all three reports. Few studies reported direct cardiac reprogramming in other species such as rats, pigs and guinea pigs (Table 4). 


\begin{tabular}{|c|c|c|c|c|c|c|c|c|c|}
\hline Starting cells & $\begin{array}{l}\text { Target } \\
\text { cells }\end{array}$ & $\begin{array}{l}\text { Rep. } \\
\text { factors }\end{array}$ & $\begin{array}{l}\text { Additional } \\
\text { factors }\end{array}$ & $\begin{array}{l}\text { Expression } \\
\text { system }\end{array}$ & $\begin{array}{c}\text { In } \\
\text { vivo/ } \\
\text { in } \\
\text { vitro }\end{array}$ & Functional assay & Rep. time & Efficiency & Ref. \\
\hline $\begin{array}{l}\text { ESC-fetal } \\
\text { heart-, neonatal } \\
\text { skin- derived } \\
\text { fibroblasts }\end{array}$ & CMs & GMTEM & $\begin{array}{l}\text { MYOCD, } \\
\text { ZFPM2, } \\
\text { TGF } \beta\end{array}$ & Retrovirus & $\begin{array}{l}\text { In } \\
\text { vitro }\end{array}$ & $\begin{array}{l}\mathrm{Ca}^{2+} \\
\text { flux and action potentials }\end{array}$ & No beating & $\begin{array}{c}13 \% \mathrm{MHC}+/ \mathrm{cTnT}+ \\
\text { cells (after } 2 \text { weeks) }\end{array}$ & {$[60]$} \\
\hline HDFs & CPCs & HGMT & $\begin{array}{l}\text { BMP4, Activin } \\
\text { A, bFGF }\end{array}$ & Proteins & $\begin{array}{l}\text { In } \\
\text { vitro }\end{array}$ & $\begin{array}{l}\text { Colony formation, } \\
\text { differentiation into three } \\
\text { cardiac lineages, } \mathrm{Ca}^{2+} \\
\text { transients and beating } \\
\text { (after CM differentiation), } \\
\text { transplantation }\end{array}$ & $\begin{array}{l}20 \text { days } \\
\text { (from } \mathrm{CPCs} \\
\text { to } \mathrm{CMs} \text { ) }\end{array}$ & $\begin{array}{c}81 \% \text { Flk } 1+, 83 \% \\
\text { Isl1+ } \\
\text { (after } 8 \text { days) }\end{array}$ & [65] \\
\hline HDFs, HCFs & CMs & GMTMM & & $\begin{array}{l}\text { Retrovirus, } \\
\text { lentiviruses }\end{array}$ & $\begin{array}{l}\text { In } \\
\text { vitro }\end{array}$ & $\begin{array}{c}\text { Action potentials, } \\
\text { beating, } \mathrm{Ca}^{2+} \\
\text { transients (after co- } \\
\text { culture with neonatal rat } \\
\text { CM) }\end{array}$ & 2 weeks & $\begin{array}{c}5 \% \text { a-actinin }+, 5 \% \\
\text { cTnT+ cells (after } 4 \\
\text { weeks) }\end{array}$ & [61] \\
\hline HDFs & $\begin{array}{l}\mathrm{CPCs} \text {, } \\
\mathrm{CMs}\end{array}$ & EM & $\begin{array}{l}\text { Activin A, } \\
\text { BMP2 }\end{array}$ & $\begin{array}{l}\text { Proteins, } \\
\text { lentiviruses }\end{array}$ & $\begin{array}{l}\text { In } \\
\text { vitro }\end{array}$ & $\begin{array}{c}\text { Beating, } \mathrm{Ca}^{2+} \\
\text { transients }\end{array}$ & 3 weeks & $\begin{array}{c}8 \% \text { CD } 31+/ K D R+ \\
\text { cells }(7 \text { days) } \\
17 \% \text { MHC+ cells, } \\
8 \% \text { beating cells }(3 \\
\text { weeks) }\end{array}$ & [66] \\
\hline HDFs & $\begin{array}{l}\text { c-Kit+ } \\
\text { CPCs }\end{array}$ & HGMT & & mRNAs & $\begin{array}{c}\text { In } \\
\text { vitro }\end{array}$ & $\begin{array}{l}\text { Differentiation to CMs } \\
(20 \% \text { cTnT + cells })\end{array}$ & No beating & $4.9 \%$ c-Kit+ cells & [67] \\
\hline $\begin{array}{l}\text { HFFs, AHCFs, } \\
\text { AHDFs }\end{array}$ & $\mathrm{CMs}$ & HGMT2m & & Retroviruses & $\begin{array}{l}\text { In } \\
\text { vitro }\end{array}$ & $\begin{array}{l}\text { Beating, calcium } \\
\text { transient }\end{array}$ & 11 weeks & $\begin{array}{c}14 \% \text { cTnT+ cells } \\
\text { (AHCFs, } 2 \text { weeks), } \\
19 \% \text { cTnT+ cells } \\
\text { (HFFs, } 5 \text { weeks) }\end{array}$ & [62] \\
\hline HCFs & CMs & GMTMM & miR-133 & $\begin{array}{c}\text { Retrovirus (GTM), } \\
\text { synthetic mimics } \\
\text { miR133 }\end{array}$ & $\begin{array}{l}\text { In } \\
\text { vitro }\end{array}$ & 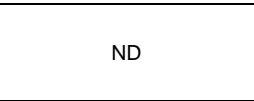 & ND & $\begin{array}{c}23 \%-27 \% \text { cTnT+ } \\
\text { cells, } \\
8 \% \text { a-actinin cells } \\
\text { (after } 1 \text { week) }\end{array}$ & {$[55]$} \\
\hline
\end{tabular}

\section{Direct cardiac reprogramming in vivo}

Few years before the first report on direct cardiac reprogramming was published, Zhou et al. observed that adult pancreatic exocrine cells could be reprogrammed to beta-cells in vivo [16], which indicated that some degree of plasticity existed between closely related cell types in the organs. The ultimate goal of direct reprogramming was to harness the endogenous cells within an organ to restore normal structure and function, without requiring the use of cell-based therapy. CFs, found throughout cardiac tissue, accounting for up to $50 \%$ of all cells in the adult heart [68]. Unfortunately, these abundant cells don't have any specific markers, although markers such as discoidin domain receptor tyrosine kinase 2 (DDR2), fibroblast-specific protein (FSP), vimentin and periostin are widely used by scientists to identify CFs, as well as for lineage-tracing studies [69]. Relatively poor quality and low efficiency of in vitro cardiac reprogramming might 
possibly be attributed to the lack of a natural condition for CMs in vitro. However, we have shown, along with other researchers, that CFs derive from different origins (epicardium, endothelial cells, and Pax3-expressing cells), which might respond differently during in vivo reprogramming $[70,71]$.

In 2012, two years after reporting GMT as cardiac reprogramming factors, pioneer scientists in cardiac reprogramming published four reports (Figure 2, Table 2) that demonstrated in situ reprogramming of CFs to CM-like cells that contributed in heart regeneration $[32,45,49,53]$. Srivastava and colleagues reported that local delivery of retroviruses expressing Gata4, Mef2C, and Tbx5 after coronary ligation resulted in the emergence of new binucleated iCMs that assembled sarcomeres and had CM-like gene expression (Figure 1, Table 2) [45]. Although in vivo cardiac reprogramming occurred with a similar initial efficiency as observed in vitro (10\%$15 \%$ ), the in vivo iCMs were mature and more closely resembled endogenous CMs than their cultured counterparts did. These researchers showed that iCMs decreased scar size and improved heart function. In addition, co-administration of thymosin $\beta 4$ along with GMT enhanced GMTmediated regeneration and further increased heart function (ejection fraction). Ieda and colleagues simultaneously showed that GMT could reprogram CFs to iCMs in vivo (Figure 2, Table 2) [49]. They showed that the polycistronic vector expressing GTM more efficiently transduced CFs and generated more mature iCMs compared to three separate vectors. On the other hand, Olson and colleagues found that HGMT could cooperatively reprogram dividing non-CM cells into functional CM-like cells, which improved cardiac function and reduced adverse ventricular remodeling following an MI (Figure 2, Table 2) [32]. The last report published in 2012 by Dzau and colleagues discussed the combination of 4 miRNAs as an "miRNA combo" (miRNA-1, miRNA-133, miRNA-208, and miRNA-499) that had the 
capability to induce direct cellular reprogramming of fibroblasts to CM-like cells in vitro and in vivo (Figure 2, Table 2) [53]. They observed that $12 \%$ of CMs at the infarct border zone comprised newly generated iCMs. In their study, iCMs expressed CM markers, sarcomere organization, excitation-contraction coupling, and action potential characteristic of mature ventricular cardiac myocytes at 5 to 6 weeks after the injury.

These and numerous other researchers further improved the efficiency of the original research (discussed below). For example, it has been shown that prevascularization of the infarcted region of a rat heart with adenovirus that encodes all three major isoforms of vascular endothelial growth factor (VEGF) potentiates the effect of reprogramming with GMT, which results in greater improvements in postinfarct myocardial function (Table 4) [72].

On the other hand, few reports demonstrate that in situ CMs can be reprogrammed to specialized non-contracting myocytes called pacemaker cells (Table 4). For example, overexpression of Tbx18 in the hearts of pigs and guinea pigs apparently reprograms CMs into pacemaker-like cells, which could be a potential alternative to mechanical pacemakers (Table 4)

Table 4. Direct cardiac reprogramming in other species (rats, pigs, guinea pigs, and canines)

\begin{tabular}{|c|c|c|c|c|c|c|c|c|c|}
\hline Starting cells & $\begin{array}{l}\text { Target } \\
\text { cells }\end{array}$ & $\begin{array}{l}\text { Rep. } \\
\text { factors }\end{array}$ & $\begin{array}{l}\text { Additional } \\
\text { factors }\end{array}$ & $\begin{array}{l}\text { Expression } \\
\text { system }\end{array}$ & $\begin{array}{c}\text { In vivo } \\
/ \\
\text { in vitro }\end{array}$ & Functional assay & $\begin{array}{l}\text { Rep. } \\
\text { Time }\end{array}$ & Efficiency & Ref. \\
\hline $\begin{array}{c}\text { CFs and skin fibroblasts } \\
\text { (canine) }\end{array}$ & $\mathrm{CMs}$ & HGMT & & Lentivirus & In vitro & $\begin{array}{c}\text { Cell } \\
\text { transplantation }\end{array}$ & ND & $\begin{array}{c}\begin{array}{c}12 \%-17 \% \text { Tnnt } 2+ \\
\text { cells } \\
\text { (after } 2 \text { weeks ) }\end{array} \\
\end{array}$ & [73] \\
\hline $\begin{array}{l}\text { CMs } \\
\text { (pig) }\end{array}$ & PCs & Tbx18 & & Adenovirus & In vivo & ECG & $\begin{array}{c}2 \\
\text { weeks }\end{array}$ & ND & {$[74]$} \\
\hline $\begin{array}{c}\text { Neonatal rat ventricular } \\
\text { myocytes } \\
\text { (guinea pigs \& rats) }\end{array}$ & PCs & Tbx18 & & Adenovirus & $\begin{array}{c}\text { In vitro } \\
\text { (rat) } \\
\text { in vivo } \\
\text { (guinea } \\
\text { pig) }\end{array}$ & Automaticity & $\begin{array}{c}2 \\
\text { days }\end{array}$ & $\begin{array}{l}9.2 \% \\
\text { (in vivo) }\end{array}$ & {$[75]$} \\
\hline $\begin{array}{l}\text { CFs (in vitro), scar CFs } \\
\text { (in vivo) } \\
\text { (rat) }\end{array}$ & CMs & $\begin{array}{l}\text { Polycistronic } \\
\text { GMT }\end{array}$ & $\begin{array}{l}\text { Pre- } \\
\text { conditioning } \\
\text { with VEGF }\end{array}$ & Lentivirus & $\begin{array}{l}\text { In vitro, } \\
\text { in vivo }\end{array}$ & $\begin{array}{l}\text { Improvement in } \\
\text { ejection } \\
\text { fraction and } \\
\text { fractional } \\
\text { shortening }\end{array}$ & ND & $\begin{array}{l}7.5 \% \text { cTnT+ cells } \\
\text { (in vitro, } 2 \text { weeks) }\end{array}$ & {$[76]$} \\
\hline $\begin{array}{l}\text { Dermal fibroblasts, in } \\
\text { situ scar CFs } \\
\text { (rat) }\end{array}$ & CMs & GMT & $\begin{array}{c}\text { Pre- } \\
\text { conditioning } \\
\text { with VEGF }\end{array}$ & Lentivirus & $\begin{array}{l}\text { In vivo, } \\
\text { in vitro }\end{array}$ & $\begin{array}{l}\text { Echocardiographic } \\
\text { analysis (for in } \\
\text { vivo) }\end{array}$ & ND & $\begin{array}{l}7 \% \text { cTnT+ cells } \\
\text { (in vitro, after } 2 \\
\text { weeks) }\end{array}$ & [72] \\
\hline
\end{tabular}




\section{Boosters of direct cardiac reprogramming}

It is well-known that numerous different factors (signaling pathways, TFs, epigenetic remodeling factors) affect the development and maintenance of cardiac identity, as well as increase the efficiency of iPSC generation (from fibroblasts) and CM differentiation (from pluripotent stem cells) $[77,78]$. Interestingly, some of these factors/mechanisms highly affect different aspects of direct reprogramming such as the efficiency of iCM generation, iCM maturity, and the type of iCMs (atrial, ventricular, and nodal).

\section{Hypoxia}

Oxygen is an important component of a microenvironment. Hypoxia promotes the proliferation of a variety of mammalian cell types such as ESCs, smooth muscle cells, endothelial cells, and fibroblasts [79]. Hypoxia causes a range of cardiac malformations, including myocardial and valvular hypoplasia [80]. It has been demonstrated that hypoxia, mostly via HIF-1, HIF-2 and VEGF, is involved in the development of coronary vessels, myocardial structure, and optimal cardiac performance [81]. A hypoxic culture $\left(4 \% \mathrm{O}_{2}\right.$ tension) can increase the yield of iPSCderived CM by approximately 1000-fold [82]. Most recently, Sadek and colleagues published an extremely impressive paper in Nature which demonstrated that in adult mice, gradual exposure to severe systemic hypoxemia resulted in inhibition of oxidative metabolism, decreased reactive oxygen species (ROS) production and oxidative DNA damage, and reactivation of CM mitosis and heart regeneration [83]. According to the various important roles of oxygen in $\mathrm{CM}$ generation and function, oxygen appeared to affect direct cardiac reprogramming. Recently, Wang et al. reported that a short term pretreatment of neonatal mouse dermal fibroblasts with low levels (2\%) of oxygen for $6 \mathrm{~h}$ improved the efficiency of GMT-based cardiac 
reprogramming (Table 2) [46]. They showed that hypoxic pretreatment for $6 \mathrm{~h}$ increased the efficiency of Myh6+ and cTnI+ cell generation by 10- and 2.0-fold, respectively. However, Myh6+ and cTnI+ cells remained morphologically indistinguishable from Myh6- and cTnI- cells and exhibited no spontaneous beating activity. Surprisingly, short-term hypoxia pretreatment resulted in a significant difference in CM-like cells after 4 weeks, but not earlier. In comparison with normoxic conditions, HIF-1 $\alpha$ expression slightly increased during the two first weeks and then returned to normal levels. Although these researchers did not achieve fully reprogrammed functional iCMs, their study shed light on the effect of microenvironment factors on cardiac reprogramming [46]. Very recently, it has been demonstrated that hypoxia increases genomewide epigenetic bivalency (H3K4me3 and H3K27me3) in breast cancer [84]. Hypoxia rapidly increases $\mathrm{H} 3 \mathrm{~K} 4 \mathrm{me} 3$ and $\mathrm{H} 3 \mathrm{~K} 27 \mathrm{me} 3$ marks at specific locations throughout the genome and are largely reversed upon reoxygenation. It has been shown that H3K27me3 demethylase KDM6B/JMJD3 is inactivated by limited oxygen [84]. Hypoxia possibly enhances cardiac reprogramming via altering epigenetic modifications (especially epigenetic bivalency). It has been recently shown that bivalency is strongly involved in cardiac reprogramming (discussed later) and might work along with other cardiac reprogramming boosters, especially those that work on epigenetic modifications. An investigation into the effects of different levels of oxygen on direct reprogramming in vivo and in vitro would be of interest.

\section{Topographical cues}

Cellular organization is highly affected by components of the microenvironment such as collagen fibers, fibronectin, and interstitial cells. Similar to in vivo conditions, microenvironment properties that include plate surface topography and the type and orientation of components used 
for coating the surface can affect a cell's identity in vitro [85]. Downing et al. have shown that parallel microgrooves on the surface of cell-adhesive substrates, as well as nanofibrous scaffolds with aligned fiber orientation increased the efficiency of iPSC generation from mouse fibroblasts [86]. It has been shown that physical cues such as mechanical stretching and microgrooves positively affect stem cell differentiation to CM. In addition, they increase the maturation of neonatal CMs and PSC-derived CMs (PSC-CMs ) [87, 88]. Recently, two different groups have shown that biophysical cues affect direct cardiac reprogramming in vitro. Sia et al. studied the effects of periodic mechanical stretch, substrate stiffness, and microgrooved substrate on direct cardiac reprogramming mediated by GMT in mouse TTFs and CFs [40]. They reported that only the microgrooved substrate increased the number of $\mathrm{cTnT}+/ \alpha \mathrm{MHC}+$ cells to approximately onefold in comparison to the flat substrate (Table 2). However, they observed beating iCMs and calcium transient when CFs were used as starting cells along with an optimized protocol developed by Ieda and colleagues [52]. In their study, a microgrooved substrate significantly increased the number of beating iCMs and striated cTnT+ cells compared to the flat substrate. Mechanical stretching and substrate stiffness did not positively affect cardiac reprogramming. To find the molecular mechanism thereby microgroove enhanced direct cardiac reprogramming, they used three small molecules which influence the cytoskeletal network: blebbistatin (Bleb), an inhibitor of myosin II activity; jasplakinolide (Jasp), an activator for actin polymerization; and cytochalasin D (CytoD), an inhibitor for actin polymerization. Interestingly, Bleb reduced the amount of iCM on the microgrooved surface compared to the flat surface, whereas Jasp and CytoD increased the yield of iCMs on the flat surface compared to the microgrooved surface. They have shown that the effects of the microgroove were mediated by the sub-cellular localization and activity of Mkl1 [also known as myocardin related transcription factor-A (Mrtf- 
A)], a mechanosensitive TF that binds to G-actin and sequesters in the cytoplasm. In their study, Bleb reduced the yield of iCMs by sequestering Mkl1 in the cytoplasm, whereas Jasp and CytoD improved the yield of $\mathrm{iCMs}$ by translocating Mkl1 into the nucleus. However, Mkl1 overexpression only partially recapitulated the effect of the microgroove and its effect was slightly improved by valproic acid (VPA). Their results indicated that VPA and Mkl1 overexpression could only recapitulate the effect of the microgroove in the earlier stage or reprogramming by day 10 , but not over the long term as measured by the number of beating cells and striated cTnT+ cells. This finding indicated that the microgroove had an additional effect in the later stage of reprogramming on the maturation and organization of the sarcomeric structure. Other studies demonstrated that Mkl1 promoted cardiac reprograming in combination with other TFs. The addition of Mkl1 or myocardin to HGMT significantly increased the number of tropomyosin+ and cTnT+ cells from HFFs [62].

In the other study, Morez et al. reported that parallel microgrooves could effect on differentiation/reprogramming of Lin-/Sca1+ cardiac side population (SP) cells into CM-like cells [89]. The microgrooved substrate caused the cells to adopt a very elongated morphology with dramatic nuclear elongation. This substrate decreased cell proliferation by $25 \%$, but lacked the capability to significantly induce cardiac differentiation. Unlike previous studies, parallel microgrooves did not increase histone 3 acetylation ( $\mathrm{AcH} 3)$ in all studied cells, suggesting that topographically induced hyper-acetylation on microgrooved substrates was not ubiquitous and might depend on the cells' baseline acetylation levels. However, a direct correlation existed between the nuclear elongation and $\mathrm{AcH} 3$ levels. Microgrooves enhanced direct cardiac reprogramming by 2 -fold in cardiac progenitors with overexpressed Mef2C, myocardin, and Tbx5. This enhancement was observed in cells with hyper-acetylation induced by the 
microgrooved substrate. In support of other studies, VPA induced a global increase in AcH3 for one of the studied cell lines (SP16), but not for another studied cell line (SP3). The expression of exogenous TFs along with VPA treatment enhanced cardiac reprogramming on a flat substrate. Interestingly, microgrooved substrates promoted myocardin sumoylation, which has been proven to strongly activate cardiogenic gene activity. Consistent with another study, the researchers observed the development of highly ordered sarcomeres on the microgrooved substrate with a 10 -fold increase in the number of $\alpha \mathrm{MHC}+$ cells compared to the flat surface. These two studies demonstrated a relationship between the biophysical microenvironment, epigenetic mechanomodulation, and direct cardiac reprogramming [40, 89]. Probably, specific topographical cues could enhance the maturation of human and mouse iCMs derived using different factors and strategies.

\section{Stoichiometry}

It is very important to note that during cardiac development the dosage of signaling pathways, morphogens, TFs, and epigenetic regulating factors are very essential for normal cardiac development. For example, retinoic acid has dose-dependent, time-sensitive, sequential, and multifaceted roles that regulate different aspects of cardiac development such as atrialventricular identity [90]. On the other hand, most mutations that cause cardiac diseases appear to result in haploinsufficiency. For example, in crosses between Tbx5, Nkx2.5, and Gata4 mutant mice, these factors genetically interacted, and compound heterozygosity of any two genes leads to far more severe heart disease than haploinsufficiency of single genes [77], indicating that TF interactions are stoichiometrically regulated. Moreover, the haploinsufficiency of $\operatorname{Brg} 1$, the central catalytic subunit of numerous chromatin-modifying complexes, is considerably worse 
when combined with haploinsufficiency of cardiac TFs (Tbx5, Nkx2.5, or Tbx20) [91]. This indicates a strong stoichiometric relationship between TFs and/or chromatin-remodeling complexes.

Recently, Munshi and colleagues showed that reprogramming of mouse fibroblasts with HGMT generated diverse types of CMs [44]. They used Hcn4-GFP fibroblast as starting cells and showed HGMT that resulted in 3 different cell types that included 35\% induced atrial-like myocytes (Hcn4-GFP-/ $\alpha$-actinin+/Nppa+ cells), 20\% induced ventricular-like myocytes (Hcn4GFP-/ $\alpha$-actinin+/Myl2+ cells), and 32\% induced pacemaker-like myocytes (Hcn4-GFP+/ $\alpha$ actinin+/Nppa-cells) (Table 2). Since they used four factors, the heterogeneous fate of GHMTreprogrammed fibroblasts might reflect the particular combination and dosage of TF expressed in a given cell. On the other hand, Dixon and colleagues analyzed the individual and combined roles of Nkx2.5, Tbx5, Gata4, and Myocd in activating cardiac gene expression [36]. They found that Gata4 alone inhibited genes involved in non-cardiac processes. Myocd alone activated genes involved in a number of cardiac-related processes and inhibited genes involved in the Wnt receptor signaling pathway and non-cardiac processes. Tbx5 alone activated genes involved in the Wnt receptor signaling pathway and inhibited genes involved in a number of cardiac-related processes. Interestingly, the combination of Tbx5 (T), Gata4 (G), and Myocd (My) was the most effective activator of genes associated with cardiac-related processes. Unlike T, My or G alone or the combination of $\mathrm{T}+\mathrm{My}$, the researchers observed that the combination of $\mathrm{T}+\mathrm{G}+\mathrm{M}$ activated the fewest genes associated with non-cardiac processes. Their results indicated that the combinations of $\mathrm{T}+\mathrm{My}$ and $\mathrm{T}+\mathrm{G}+\mathrm{My}$ only had the capability to induce cardiac marker protein expression. These observations indicated that the mixture of viruses which expressed individual factors resulted in heterogeneous and uncontrollable ratios of reprogramming factor expression 
among infected fibroblasts, which might lead to variable and low reprogramming efficiency. It is mathematically reasonable that the rate of infected cells decreases with increasing numbers of exogenous factors. Usually the rate of infected cells is approximately $90 \%$, as determined by GFP expression. Therefore, in triple infections, only $72.9 \%$ of cells have been transduced by all three factors as calculated by $(0.90 \times 0.90 \times 0.90) \times 100$. Polycistronic vectors can increase the chance of receiving all factors by starting cells. Reports indicate that polycistronic vectors that express GMT increase the efficiency of cardiac reprogramming in vitro and in vivo. For example, Ieda and colleagues designed a polycistronic retrovirus that expressed GMT separated by $2 \mathrm{~A}$ self-cleaving peptides (3F2A) in an attempt to transduce GMT efficiently in vivo [49]. They showed that the 3F2A-induced CM-like cells in fibrotic tissue expressed sarcomeric $\alpha$-actinin and cTnT, and had clear cross striations (Table 2). Rosengart and colleagues reported that the efficiency of cardiac reprogramming mediated by a polycistronic vector expressing GMT was double compared to singlet G, M, T system [76]. However, the polycistronic system resulted in expression of a homogeneous ratio of all factors in the infected cells. Clearly, stoichiometry was very important in iPSC generation. Elevated expressions of Oct4 and Klf4 combined with lower expressions of c-Myc and Sox2 efficiently produced iPSCs [92]. In order to test the stoichiometry in direct cardiac reprogramming, Qian and colleagues generated a complete set of polycistronic constructs (MGT, MTG, GMT, GTM, TMG, and TGM) with all possible splicing orders and analyzed the cardiac reprogramming efficiency using these vectors [47]. They found that each polycistronic vector gave rise to distinct $\mathrm{G}, \mathrm{M}$, and $\mathrm{T}$ protein expression levels. Interestingly, only MGT and MTG enhanced cardiac reprogramming compared to the separate $\mathrm{G}, \mathrm{M}$, and $\mathrm{T}$ vectors, indicating high levels of Mef2C and low levels of Gata4 and Tbx5 are required for efficient cardiac reprogramming. Importantly, after further optimization, the MGT 
vector resulted in more than a 10 -fold increase in the number of mature beating iCM loci. Molecular characterization revealed that the optimal stoichiometry caused higher expression of mature cardiac myocyte markers. Interestingly, the expression of fibroblast markers (Col1a1, Col3a1, and elastin) significantly decreased with all 6 constructs, which suggested that they exhibited a similar capacity to repress gene expression of fibroblast markers. Qian and colleagues also used their optimal polycistronic vector for in vivo cardiac reprogramming [50]. Importantly, polycistronic MGT-treated mice exhibited a further improvement in heart function compared with the singlet G, M , and T-treated group. Polycistronic MGT caused a further reduction in scar size compared with the singlet G, M, T group. They found that both polycistronic MGT and G, M, T resulted in similar cardiac reprogramming quality in vivo [50]. These results suggest, in general, that the stoichiometry of TFs is critical for cellular reprogramming. This finding is consistent with the idea that precise dosage and temporal expression of TFs are important for cell fate specification and development. Stoichiometry can be tested for other reported combinations of mouse or human fibroblasts. Direct cardiac reprogramming of human fibroblasts will be more efficient if the reprogramming TFs or miRNAs are stoichiometrically administrated. Many different combinations claim to be the most efficient for mouse fibroblast reprogramming, however polycistronic MGT is becoming the universal, most optimal combination for in vivo and in vitro cardiac reprogramming of mouse fibroblasts.

\section{VEGF and FGFs (PI3K/Akt signaling pathway)}

VEGF and fibroblast growth factors (FGFs) are secreted signaling proteins that play important roles during cardiac development and disease. For example, FGF8, FGF9, FGF10, and FGF16 
act as paracrine signals in embryonic heart development, whereas FGF2 and FGF10 play roles as paracrine signals in postnatal heart pathophysiology and stimulate the cardiac differentiation of cultured stem cells [93].VEGF and FGFs exert their roles through several mechanisms such as p38 mitogen-activated protein kinase ( p38 MAPK) and the phosphoinositide 3-kinaseserine/threonine kinase A (PI3K/Akt pathway). The PI3K/Akt pathway regulates numerous aspects of cardiology, including CM differentiation and survival, myocardial hyperplasia and hypertrophy, myocardial contractility, electrophysiology, and energy metabolism [94].

Recently, Olson and colleagues reported that overexpression of constitutively activated Akt1 along with HGMT enhanced the efficiency of direct cardiac reprogramming from various types of mouse fibroblasts (MEFs, TTFs, CFs) [37]. They found that addition of Akt1 to HGMT significantly increased the number of iCMs that displayed higher maturity and functionality as shown by an altered metabolic profile, increased ratio of Myh6:Myh7 expression, increased cell size, change in morphology, emerging multi/binucleated iCMs, and mitochondrial membrane depolarization activity. For the first time, they showed the production of multinucleated iCMs in vitro, which increased in the presence of Akt1. These researchers also determined that IGF1 increased the efficiency of reprogramming, although activation of Akt1 by IGF1 was not as potent as overexpressed constitutively activated Akt1. They also observed that the small molecule LY294002, an inhibitor for PI3K, reduced direct cardiac reprogramming efficiency. Inhibition of mTORC1 by rapamycin and activation of Foxo3a by genetic addition impaired direct cardiac reprogramming induced by HGMT plus Akt1. These results have indicated that IGF1 receptor and PI3K are the upstream mediators and mTORC1 and Foxo3a are the downstream mediators of Akt1 in boosting direct cardiac reprogramming from mouse fibroblasts [37]. Lien et al. described mechanisms by which the PI3K-Akt-mTOR network controlled 
cellular metabolism in cancer [95]. The PI3K-Akt-mTOR pathway coordinated the uptake and utilization of multiple nutrients, including glucose, glutamine, nucleotides, and lipids. Metabolism reprogramming using manipulation of this signaling might efficiently improve the rate and quality of cardiac reprogramming. Srivastava and colleagues reported a role for Akt1 in enhancing direct cardiac reprogramming in vivo [45]. They administrated thymosin $\beta 4$ along with GMT transduction into a mouse heart after MI. Coinjection of thymosin $\beta 4$ and GMT yielded further functional improvement in ejection fraction and cardiac output, and reduced the scar size compared to either thymosin $\beta 4$ or GMT injection alone (Table 2). Thymosin $\beta 4$ has been shown to activate Akt1. Thymosin $\beta 4$, probably via Akt1, increased the efficiency of iCMs through at least two mechanisms: 1) promotion of CFs migration from intact sites or from the epicardium to the infarcted area and 2) enhancement of CFs proliferation. These two mechanisms increased the number of CFs that could be infected by GMT, which resulted in an increased percentage of iCMs [45]. However, Olson and colleagues showed that the proliferation of MEFs and iCMs reduced even in the presence of Akt1. They did not observe any difference in apoptosis between cells treated with HGMT and HGMT plus Akt1. Therefore, it seems that Akt1 might work via different mechanisms in vivo and in vitro. In another study, Ieda and colleagues reported a stepwise, efficient method for direct reprogramming of mouse fibroblasts to iCMs [52]. They screened eight cardiogenic compounds and found that the combination of FGF2, FGF10, and VEGF (FFV) increased the efficiency of cardiac reprogramming. Under optimized culture conditions, they cultured GMT-transduced cells for one or two weeks in FBS that contained media followed by two weeks' culture with serum-free medium plus FFV, which they named FFV. This stepwise reprogramming strategy greatly increased the efficiency of cardiac reprogramming as determined by higher numbers of beating iCMs and spontaneous $\mathrm{Ca}^{2+}$ 
oscillations. They did not observe any significant effects from the other factors (Activin A, BMP4, CHIR99021, DKK1, and TGF $\beta$ ). Interestingly, FFV led to significantly improved cardiac reprogramming compared to that observed with the addition of only one or two growth factors in serum-free medium. Treatment with FFV for the last 2 weeks adequately enhanced cardiac reprogramming, whereas FFV treatment for the first week did not affect reprogramming, which suggested that FFV was critical for the late but not early stage of reprogramming. FFV increased the number of beating iCMs by conversion of partially reprogrammed iCMs into functional iCMs at the late stage of reprogramming. In this study, the researchers demonstrated that the higher efficiency was not due to the generation of partially reprogrammed iCMs or mitotic CPCs, proliferation of iCMs, or induction of pacemaker cells. Interestingly, microarray analysis indicated that FFV promoted cardiac reprogramming by activation of cardiac programming rather than suppression of fibroblast signatures. They also found that cardiac reprogramming efficiency further increased by the addition of IWP4, an inhibitor of Wnt signaling, to the FFVcontaining medium. Their results indicated that the FFV treatment strongly induced expressions of phospho-ERK1/2, phospho-p38MAPK, and phospho-AKT. On the other hand, SB203580 (p38MAPK inhibitor) and LY294002 (PI3K inhibitor), but not PD98059 (MEK inhibitor), reduced the generation of beating iCMs, which suggested that FFV improved cardiac reprogramming through p38MAPK and PI3K/AKT, but not through MEK/ERK1/2. Interestingly, they found that FFV could replace Gata4; however, the efficiency of cardiac reprogramming by Mef2C and Tbx5 plus FFV was significantly lower than GMT plus FFV. Importantly, FFV enhanced cardiac reprogramming mediated with other combinations such as HGMT or GMT plus Mesp1 and Myocd (GMTMM) even in refractory cells such as TTFs, which suggested that FFV could increase the efficiency of any type of combination [52]. 
It has been reported that VEGF increased the efficiency of cardiac reprogramming in vivo [76]. Rosengart and colleagues induced MI in rats and intramyocardially administrated an adenovirus encoding all 3 major isoforms of VEGF (AdVEGF), followed 3 weeks later by the administration of individual $\mathrm{G}, \mathrm{M}$, and $\mathrm{T}$ or a polycistronic GMT. They showed that administration of individual G, M, and T induced postinfarct ventricular functional improvement and that polycistronic vector GMT enhanced the efficacy of this strategy. However, they did not show how VEGF played a role in the in vivo cardiac reprogramming. Previous studies predicted roles for VEGF in the heart. For example, VEGF-B induced an expanded coronary arterial tree (via Erk1/2) and reprogramming of CM metabolism (via Akt and mTORC1). VEGF-B overexpression in hearts have been shown to shift from fatty acid to glucose oxidation pathways [96].

\section{BMP4 and Activin A}

BMPs and Activin A are subgroups of the TGF $\beta$ superfamily. BMPs appear to have a role in expansion of cardiac progenitors, as genetic deletion of the BMP receptor in Mesp1-expressing mesoderm results in major defects in heart formation [97]. In cardiac progenitors, BMPs maintain the balance between precursor expansion and differentiation. Overexpression of Activin has been shown to activate heart formation in both avian and amphibian embryos. Mouse ESCs lacking the Activin co-receptor, Cripto, fail to differentiate into CM [98]. In particular, addition of a growth factor cocktail that contains Activin A and BMP4 to differentiating human ESCs results in the expansion of a cardiac progenitor population [2].

Recently, two groups used these growth factors to reprogram human fibroblast to CPCs. Islas et al. showed that ETS2 and MESP1 transdifferentiated HDFs into replicative cells that expressed 
the cardiac mesoderm marker KDR [66]. They showed that for efficient derivation of cardiac progenitors, ETS2 and MESP1 should be overexpressed for 4 days followed by 2 days of treatment with BMP4 and Activin A. In the other study, Li et al. reported that HDFs could be reprogrammed to CPCs via protein transduction of HAND2, GATA4, MEF2C, and TBX5 [99]. These researchers also used BMP4 and Activin A as cardioinductive growth factors and basic FGF (bFGF) as a maintenance factor of CPCs. The results indicated that protein-induced CPCs (piCPCs) are efficiently and rapidly generated under HGMT plus BMP4 and Activin A. Expectedly, the piCPCs had the capability to differentiate into three cardiac lineages: CMs, endothelial cells, and smooth muscle cells.

\section{Wnt and JAK/STAT signaling}

Wnt/ $\beta$-catenin signaling plays essential roles during vertebrate heart development. Several studies have uncovered an inhibitory role of $\mathrm{Wnt} / \beta$-catenin signaling in vertebrate heart specification, which is most dramatically shown by the formation of ectopic hearts after conditional inactivation of $\beta$-catenin in the definitive endoderm of the mouse embryo [100]. Our laboratory, along with other researchers, have demonstrated that this pathway induces cardiac specification during early developmental stages, but inhibits it later [2]. Thus, treatment of early differentiating mouse or hESC cultures with Wnt ligands enhances CM formation via promotion of mesoderm specification, while later treatment with Wnts suppresses CM formation. The Wnt inhibitor Dickkopf1 (Dkk1) promotes CM differentiation [100].

Recently, Srivastava and colleagues [59] found that a combination of the inhibitors of TGF $\beta$ (SB431542 or SB) and WNT (XAV) increased reprogramming efficiency 8-fold when added to CFs infected by polycistronic MGT [50]. The small-molecules also enhanced both the speed and 
quality of cell reprogramming, as there were beating cells as early as 1 week after reprogramming compared to 6-8 weeks with GMT alone. These two chemicals were also effective for in vivo cardiac reprogramming. They showed that mice exposed to GMT, SB, and XAV for 2 weeks after MI resulted in significantly improved reprogramming and cardiac function compared to those exposed only to GMT. Importantly, human cardiac reprogramming was equally boosted upon inhibition of TGF $\beta$ and WNT, which was most efficiently achieved by GMT plus Myocardin. They also found that 7F2i (GMT, ESRRG, MESP1, MYOCD and ZFPM2 plus SB and XAV) doubled the percentage of iCMs reprogrammed by 7F alone. Interestingly, MESP1, ZFPM2, and ESRRG were dispensable in the presence of TGF $\beta$ and Wnt inhibition as determined by a similar degree of gene expression change with just 4F2i (GMT and Myocardin plus chemicals) [59].

Canonical Wnt signaling is critical for proliferation of CPCs [2]. JAK/STAT signaling is important for normal cardiogenesis [101]. Wnt and JAK-STAT signaling have positive roles for expansion of induced CPCs or iCPCs [102]. Recently, Kamp and colleagues reported that adult mouse CFs, TTFs and lung fibroblasts could be reprogrammed into iCPCs by overexpression of 11 cardiac TFs (Mesp1, Mesp2, Gata4, Gata6, Baf60C, SRF, Is11, Nkx2.5, Irx4, Tbx5, and Tbx20). They showed that 4 days after TF expression (mediated by Dox), NKX2.5-YFP+ cells were emerged, which by 3 weeks, they developed two-dimensional, highly proliferative colonies. They showed that the combination of leukemia inhibitory factor (LIF) with BIO, a GSK3 $\beta$ inhibitor, produced the brightest EYFP+ cells that were robustly expandable. They demonstrated that LIF+BIO had a greater effect on maintenance of the reprogrammed state than on initiation of reprogramming. They found that GMT plus Baf60C and NKX2.5 (GMTNB) produced the most expandable colonies post-dox withdrawal. The iCPCs homogenously expressed Cxcr4 and 
differentiated into CMs, smooth muscle cells, and endothelial cells. Injected iCPCs could generate myocardium in the embryonic and adult post-MI mouse heart [102]. Although JAKSTAT has a positive role on proliferation of iCPCs, its role in cardiac reprogramming remains controversial. Interestingly, Dzau and colleagues found that miRNA-mediated reprogramming enhanced 10-fold upon treatment with JAK inhibitor I [53]. Remarkably, in the presence of JAK inhibitor I, miR-1 alone adequately reprogrammed the CFs. Moreover, Christoforou et al. reported that inhibition of JAK significantly enhanced the cardio-inducing effect of $7 \mathrm{~F}$ (Myocardin, SRF, Mesp1, Baf60C, Mef2C, Gata4, Tbx5) in MEFs and its effect increased more by VPA [51]. However, VPA without JAK inhibitor significantly improved the overall cardioinducing effect of 7F by approximately two-fold [51]. In contrast, Ieda and colleagues did not observe any positive effect of JAK inhibitor I on increasing the efficiency of direct reprogramming mediated by GMT plus miR combo [55].

\section{Selenium}

Dzau and colleagues developed a serum-free reprogramming median (RM) that contained supplements such as ITS, AlbuMAXII, BSA, and ascorbic acid which significantly amplified the effect of the miR combo in cardiac reprogramming of neonatal TTFs [57]. RM specifically increased expression of the cardiac gene, but not endodermal, ectodermal, and vascular genes. Surprisingly, RM in the absence of the miR combo simultaneously induced the following cardiac and pluripotency related genes: Nanog (100-fold), Oct4 ( 40-fold), Sox2 ( 30-fold), and Klf4 ( 25 -fold). The results indicated that cells cultured in RM could generate iPSC-like colonies

after culture under miPSC conditions. Surprisingly, the miR combo significantly increased the effect of RM on Nanog expression. However, the expressions of cardiac (Hand2, Tbx5, and 
Mesp1) and pluripotency genes (Oct4 and Sox2) significantly reduced following Nanog knockdown. After removal of the RM supplements, they found that selenium was the most effective component in RM that induced cardiac gene expression. Its effects were enhanced by the miR combo. Although, they did not decipher the mechanism of selenium during cardiac reprogramming, it has already been reported that selenium is an important factor for ESC expansion and pluripotency [103]. Selenium exists in a number of proteins called selenoproteins. MyoD activates the selenoprotein $\mathrm{W}(\mathrm{Se}-\mathrm{W})$ that subsequently promotes skeletal muscle differentiation. Today, several cardiac related selenoproteins have been identified such as GPx1 and TrxR2. The ablation of GPx1 and TrxR2 causes an increase in infarct size and fatal dilated cardiomyopathy, respectively. These findings increase the possibility that some selenoproteins may be involved in direct cardiac reprogramming induced by RM.

\section{Barriers of direct cardiac reprogramming}

Notably, during a reprogramming of fibroblasts to fully reprogrammed cells, progenitor cells, or iPSCs, only a few percent of infected cells are reprogrammed to the desired target cell. This finding suggests that endogenous or even exogenous barriers impede cellular reprogramming. Recently, a number of barriers and obstacles have been identified in direct cardiac reprogramming.

\section{Transforming growth factor $\beta(T G F \beta)$ signaling}

TGF $\beta$ family members (TGF $\beta$ isoforms, Activins, BMPs) have critical and specific roles in cardiac development and CM differentiation in ESCs, P19CL6 cells, CPCs, and c-Kit+ bone marrow cells [104]. TGF $\beta$ is expressed at high levels in the heart during both embryonic 
development and the adult life. TGF $\beta 2$ null mice exhibit multiple cardiac developmental abnormalities. TGF $\beta$ play an important role in tissue repair by promoting new synthesis and deposition of the extracellular matrix [105]. Most likely, the CM co-culture effects on differentiation described for other stem cell populations is mediated by TGF $\beta$ secreted by neonatal CMs [106].

Recently, Ifkovits et al. showed that inhibition of TGF $\beta$ signaling increased direct conversion of MEFs and ACFs into iCMs [33]. They used an inducible system to overexpress Hand2, Gata4, Mef2C, Tbx5 and Nkx2.5 (HGMTN), then treated the transduced cell after $24 \mathrm{~h}$ with five selected small molecules involved in CM differentiation or iPSC reprogramming. These small molecules were BIX01294 (a G9a histone methyltransferase inhibitor), CHIR and XAV (two Wnt signaling modulators), DMH1 (a BMP inhibitor) and SB (an Activin, Nodal, and TGF $\beta$ inhibitor). Interestingly, only SB led to increased iCM yield (5-fold as measured by Troponin TGCaMP). Cell treatment with LY364947 (LY), an inhibitor of the TGF $\beta$ but not Activin and Nodal components, increased the yield of $\mathrm{iCM}$ as efficiently as SB, which indicated that SB exerted its effects by inhibiting the TGF $\beta$ pathway. They also reported that to achieve the highest efficiency of direct cardiac reprogramming, TGF $\beta$ inhibition (using SB or LY) should occur simultaneously with HGMTN overexpression. Their results revealed that addition of TGF $\beta 1$ or TGF $\beta 2$, but not Activin A, totally blocked direct cardiac reprogramming as determined by lack of any Troponin T-GCaMP+ cells by 2 weeks. However, SB rescued the reprogramming of fibroblasts to iCMs in the presence of TGF $\beta 1$ or TGF $\beta 2$ and led to an increase in the percentage of Troponin T-GCaMP+ cells. Based on microarray data at day-3 post-transduction, they found that SB increased the efficiency of cardiac reprogramming by enhancing the expression of genes 
involved in cardiac muscle development and the cardiovascular system. In this research, SB did not induce iCM proliferation and apoptosis of non-reprogrammed MEFs [33].

In another study, Zhao et al. analyzed the transcriptome of HGMT infected MEFs [43]. They found that pro-fibrotic/ECM genes such as $\alpha \mathrm{SMA}$ and Colla1 significantly upregulated along with cardiac lineage genes within the first week of reprogramming. Surprisingly, they found that TGF $\beta$ signaling components were activated (p-Smad2) or upregulated (Tgfb2 and Tgfbr1) during the first week of reprogramming. These components were downregulated during later stages of reprogramming (day 14). Interestingly, TGF $\beta$ signaling targets such as Snail and Slug, which control the EMT downregulated within the first week. This finding indicated that TGF $\beta$ signaling activated in HGMT-infected fibroblasts during the early stages, but downregulated during later stages of cardiac reprogramming. As previously shown, miR-1 and miR-133 are involved in cardiac development and direct reprogramming; their addition to HGMT (HGMT2m) significantly increased the efficiency of direct cardiac reprogramming (from $32 \%$ to $42 \% \mathrm{cTnT}^{\text {hight }}$ cells and from 200 to 1400 beating iCMs at day 28). Interestingly, RNA-Seq analysis revealed that cardiac development genes enriched among genes upregulated in HGMT2m-infected cultures, whereas ECM related genes significantly enriched among genes downregulated in HGMT2m-infected cultures, which suggested that miR-1 and miR-133 enhanced cardiac reprogramming, at least in part, by attenuating pro-fibrotic gene expression. They showed that treatment of HGMT-infected cultures with TGF $\beta 1$ significantly reduced the number of $\mathrm{cTnT}+$ and $\alpha$-actinin + populations. Moreover, TGF $\beta 1$ treatment decreased beating iCMs in HGMT- and HGMT2m-infected cultures by 100 -fold, demonstrating that overactivation of pro-fibrotic signaling reduced reprogramming of fibroblasts into beating iCMs. Importantly, inhibiting ROCK, a key regulator of fibrosis, using Y-27632 decreased the expression of fibrotic 
gene ( $\alpha$ SMA) and significantly increased the number of beating iCMs in HGMT and HGMT2m infected MEFs. Other ROCK inhibitors such as Thiazovivin and SR-3677 also increased the number of beating iCMs, suggesting that ROCK signaling was a barrier for cardiac reprogramming. On the other hand, A83-01, a selective inhibitor of TGF $\beta$ signaling, decreased p-Smad2 levels and inhibited expressions of Fn-EDA, Col1a1 and Col3a1, as well as formation of aSMA stress fibers in HGMT-infected cells. Interestingly, A83-01 had a stronger effect in HGMT2m-infected cells, suggesting that two miRNAs and A83-01 synergistically suppressed pro-fibrotic gene expression in reprogramming cells. A83-01 significantly increased the percentages of cTnT + and $\alpha$-actinin + cells, and the number of beating iCMs in HGMT- and HGMT2m cultures. Other ALK5 inhibitors (LY-364947, SD-208, and GW788388) also enhanced cardiac reprogramming by inducing more spontaneously beating cells. No significant increase existed in the number of beating iCMs when ROCK (using Y-27632) and TGF $\beta$ signaling (using A83-01) were simultaneously inhibited, suggesting that inhibition of pro-fibrotic pathways by multiple chemicals could not further increase the reprogramming efficiency and it would be necessary to manipulate other barriers to further optimize cardiac reprogramming. Inhibition of TGF $\beta$ signaling by A83-01 also enhanced the direct reprogramming of adult mouse TTFs and CFs to cTnT+ cells and beating iCMs [43].

Recently Qian and colleagues showed that the use of an optimal reprogramming system, polycistronic MGT, in cardiac reprogramming resulted in decreased mRNA expressions of Egr2 and Egr3 through reprogramming that began from day 3 and decreased by $62 \%-86 \%$ at day 10 [107]. Egr2 and Egr3 are downstream targets of TGF $\beta$ signaling that mediate pro-fibrotic responses. It has been shown that Egr-3 overexpression adequately stimulated fibrotic gene expression, whereas deletion of Egr-3 resulted in substantially attenuated TGF $\beta$ responses [108], 
suggesting that TGF $\beta$ signaling strongly affected cardiac reprogramming via different combination of TFs.

Very recently, Srivastava and colleagues reported that TGF $\beta$ and Wnt signaling inhibition enhanced polycistronic MGT-based direct reprogramming of CFs to iCMs in vitro and in vivo [59]. They screened 5500 compounds from libraries of toxicologically tested compounds. The compounds were added to the cells $24 \mathrm{~h}$ after GMT transduction and they quantified $\alpha$-MHCGFP+ cells after 2 weeks. Interestingly, they found that the top hits included three molecules that inhibited TGF $\beta$ (SB, LDN-193189, and dexamethasone), three that inhibited Wnt signaling (XAV, IWR1, and myricetin), and one that inhibited both Wnt and TGF $\beta$ (D4476). The results indicated that SB most efficiently downregulated fibroblast genes and XAV was the most potent activator of cardiac genes at 2 weeks of reprogramming. They reported that for efficient reprogramming, TGF $\beta$ should be suppressed $24 \mathrm{~h}$ earlier than Wnt inhibition. Expectedly, inhibition of both signaling increased reprogramming efficiency (4-fold increase in $\alpha \mathrm{MHC}+/ \mathrm{cTnT}+$ cells), reduced the time required for beating initiation (1 week compared to $6-8$ weeks), and increased the quality of iCMs as determined by calcium transient and cardiac transcriptional profiles, which indicated that iCMs generated in the presence of two inhibitors were very similar to adult CMs. These two small molecules also increased the efficiency of GMT-based in vivo cardiac reprogramming following intraperitoneal injections for two weeks. Their results indicated that GMT2i improved heart function as early as one week after MI. This functional improvement persisted over 12 weeks. According to the results, TGF $\beta$ and Wnt inhibition replaced Mesp1, Zfpm2, and Esrrg [60] for cardiac reprogramming of adult human CFs. Both the efficacy of reprogramming and quality of iCMs were similar in GMTM2i (Gata4, 
Mef2C, Tbx5, and Myocd, SB and XAV) compared to 7F (Gata4, Mef2C, Tbx5, Myocardin, Mesp1, Zfpm2 and Esrrg) [59].

\section{Epithelial-to-mesenchymal transition (EMT)}

It is well-known that one of the important approaches to enhance the efficiency of iPSC generation is to suppress the mechanisms that sustain the mesenchymal state of fibroblasts. It seems reasonable that if the mesenchymal identity of fibroblasts cannot be sustained, it will be easy to change the cell fate to the desired cell type. Recently, a number of reports on direct cardiac reprogramming have indicated that inhibition of mechanisms, which promote EMT, can improve the efficiency of direct cardiac reprogramming. Ieda and colleagues reported that MiR133 promoted cardiac reprogramming by direct repression of Snai1 (a master regulator of EMT and fibrogenesis) and silencing fibroblast identity [55]. They showed that miR-133 overexpression (using $15 \mathrm{nM}$ miR-133 mimics) increased the efficiency of GMT-mediated cardiac reprogramming 7 -fold more than beating iCM and $\sim 10 \% \mathrm{cTnT}+/ \alpha \mathrm{MHC}+$ cells. This miRNA also shortened the duration to induce beating cells from 30 to 10 days compared to GMT alone. They showed that miR-133 directly repressed Snail to enhance the efficiency of cardiac reprogramming mediated by GMT or GMTMM. Moreover, miR-1 was able to increase the efficiency of reprogramming, but not as strong as miR-133. They found that miR-133 simultaneously activated cardiac gene expression and suppressed fibroblast signatures by downregulating the expression fibroblast genes such as Snai1. However, epithelial genes such as Cadherin 1 and Desmoplakin were not affected by MiR-133, which suggested that miR-133 did not induce the MET process. Expectedly, Snail knockdown suppressed fibroblast genes, upregulated cardiac gene expression, and induced more contracting iCMs with GMT 
transduction, which recapitulated the effects of miR-133 overexpression. In contrast, overexpression of Snai1 in GMT/miR-133-transduced cells maintained fibroblast signatures and inhibited generation of beating iCMs. MiR-133-mediated Snai1 repression was also critical for cardiac reprogramming in adult mice and human CFs. Silencing fibroblast signatures mediated by miR-133/Snai1 was a key molecular barrier during cardiac reprogramming. These results have suggested that Snai1 repression is critical for silencing fibroblast programs and activating the cardiac phenotype in MEFs induced with GMT/miR-133. Snai1 overexpression also inhibited both the induction of $\alpha$ MHC-GFP and cTnT expression in GMT/miR-133-transduced fibroblasts. They also showed that addition of miR-133 to GMTMM significantly increased direct reprogramming from human CFs. Snai1 mRNA expression was suppressed by GMTMM and further reduced by $40 \%$ with GMTMM/miR-133 in human cardiac reprogramming. Snai1 overexpression inhibited GMTMM/miR-133-mediated cardiac induction, which suggested that miR-133-mediated Snai1 suppression was also crucial for human cardiac reprogramming [55]. miR-133 and miR-1 have been combined with other cardiac TFs (Gata4, Hand2, T-box5, Mef2C, and myocardin) [62] or miRNAs $(1,133,208$, and 499) [53] to induce cardiac reprogramming from human fibroblasts. However, the authors did not identify the mechanisms by which miRNAs increased cardiac reprogramming. Qian and colleagues showed that during cardiac reprogramming mediated by the optimal condition (polycistronic MTG), the expression of central regulators of EMT (Sox9, Twist1, and Twist2) decreased by 60\%-85\% during the first 10 days of cardiac reprogramming [107].

\section{Bmil}


Today, it is well-known that the heart expresses many epigenetic factors, including both histone modifying proteins and chromatin remodelers. Among epigenetic factors, polycomb group (PcG) and trithorax group (TrxG) proteins are unique in maintaining repressed and activated chromatin configurations, respectively. PcG proteins function in multi-protein complexes that include PhoRC, PR-DUB and polycomb repressive complex 1 and 2 (PRC1 and PRC2) [109]. The PRC2 contains methyltransferase activity and induces trimethylation of H3K27 [110]. H3K27me3 is a well-known mark for silenced chromatin associated with the promoters and regulatory elements of PcG target genes. The PRC1 binds trimethylated H3K27 and induces chromatin compaction, thereby maintaining target chromatin regions in the silenced state [111]. PRC1 also monoubiquitinates histone $\mathrm{H} 2 \mathrm{~A}$ at lysine 119 , though the ubiquitinase activity of PRC1 appears to be dispensable for its silencing function [111]. Moreover, any fault in epigenetic regulation may lead to congenital heart disease. Cardiac TFs such as Tbx5 and Nkx2.5, have been shown to interact with histone modifying enzymes and regulate cardiac gene expression [112]. On the other hand, Ezh2 is a catalytic subunit of t PRC2 that induces di- and trimethylation of Lys27 on histone $\mathrm{H} 3$ (H3K27me2 and $\mathrm{H} 3 \mathrm{~K} 27 \mathrm{me} 3)$. It regulates the expression of genes involved in heart development and homeostasis [112]. Consistent with normal development/differentiation studies, it has been shown that cellular reprogramming from fibroblasts to iPSCs or iCMs is accompanied by profound changes in the epigenetic landscape/signature of fibroblasts and target cells (iPSCs or iCMs), in which the fibroblast specific signature is suppressed and the target cell signature is established [39].

Recently, Qian and colleagues employed a loss-of-function approach to identify epigenetic regulators of polycistronic MGT-induced direct cardiac reprogramming [39]. They used a shRNA system to investigate the role of 35 candidate components of chromatin modifying or 
remodeling complexes. Interestingly, reduced levels of Bmi1 as a major component of PRC1 significantly enhanced reprogramming of adult mouse fibroblasts to beating iCMs with a 5- and 10 -fold increase in $\alpha \mathrm{MHC}+$ and cTnT+ cells, respectively. They showed that this increase in iCM reprogramming efficiency happened only if Bmil was knocked down within 3 days of the MGT infection or 3 days prior to MGT transduction, which suggested that Bmi1 exerted its roles during the early stage of iCM reprogramming. They have found that Bmil suppressed iCM reprogramming independent of its role as a regulator of downstream targets p53, p16Ink4a, and p19Arf, all of which are involved in cell proliferation. Surprisingly, they found that Bmi1 directly bonded to a set of cardiogenic loci including Gata4, Nkx2.5, Is11, Pitx2, Tbx20, Hand2, and Smad6 to repress their expression. These Bmi1-bound cardiac loci were co-occupied with H3K4me3 (epigenetic active mark) or H3K27me3 (epigenetic repressive mark), H2AK119ub, Ring1B, and the PRC2 key component Ezh2, establishing a repressive chromatin state of these cardiac loci in fibroblasts. When they depleted Bmi1, H2AK119ub was completely removed and $\mathrm{H} 3 \mathrm{~K} 4 \mathrm{me} 3$ levels moderately increased at the cardiogenic loci, which resulted in a reduction in epigenetic repressive marks and de-repression of cardiogenic gene expression to efficiently promote iCM generation. According to their results, Bmi1 depletion replaced Gata4 in reprogramming fibroblasts into functional iCMs. However, depletion of Gata4 suppressed the cardiac reprogramming induced by MT plus shBmi1, suggested that Gata4 was one of the major downstream effectors of Bmi1 during iCM reprogramming. In order to have efficient cardiac reprogramming using MT, the researchers have proposed that first the epigenetic barrier should be eliminated by Bmi1 depletion. The role of Bmi1 in cardiac reprogramming differs from iPSC reprogramming in which Bmil enhances the efficiency of iPSC generation. Bmil knockdown also promotes iCM reprogramming in adult mouse fibroblasts and adult mouse CD31+ 
endothelial cells, which highlights Bmi1 as an important factor working as an epigenetic barrier to sustain somatic cell identity, as well as to limit cardiomyogenesis in adult mice [39]. Recently, Valiente-Alandi et al. have used Bmi1 as marker for in situ CPCs where they showed that the progeny of Bmil-expressing cells contributed to de novo CMs in infarcted hearts [113]. However, apical resection of the pups' hearts indicated a minor contribution of Bmil derived cells to de novo CMs.

\section{Ezh2 activity}

Recently Hirai et al. showed that inhibition of suppressive modifications promoted direct cardiac reprogramming in mouse fibroblasts [38]. They previously reported that fusion of M3 domain derived from MyoD to the pluripotency factor Oct 4 and Mef2C improved the efficiency of iPSC [114] and iCM generation [34], respectively. They also showed that inhibition of suppressive histone modifications (H3K27me3 and $\mathrm{H} 3 \mathrm{~K} 9 \mathrm{me} 2$ ) enhanced direct cardiac reprogramming mediated by MM3HGT. GSK126, an Ezh2 inhibitor (used from days 1 to 4), increased the number of iCM clusters and iCMs that exhibited asynchronous $\mathrm{Ca}^{2+}$ oscillation and spontaneous beating. UNC0638 significantly increased the efficiency of cardiac reprogramming. UNC0638 has been shown to inhibit two closely related enzymes, G9a and GLP, which form a heterodimer and mediate mono- and dimethylation of Lys9 on $\mathrm{H} 3$ (H3K9me and $\mathrm{H} 3 \mathrm{~K} 9 \mathrm{me} 2)$ and methylation of non-histone substrates such as p53. Interestingly, these two small molecules exert their effects on improving the efficiency of direct cardiac reprogramming at different time points (GSK126: days 1 to 4 and UNC0638: days 3 to 7). Importantly, GSK126 alone was more effective than GSK126 + UNC0638 and the combination of the two inhibitors was more effective than a single inhibitor only for HGMT. UNC0638 was more effective with HGMT than with MM3HGT. It 
has been proposed that $\mathrm{M} 3$ can bind and interact with many chromatin proteins such as histone acetyltransferases (SAGA, NuA4, p300, and PCAF), SWI/SNF chromatin remodeling ATPases, histone covalent modifying enzymes, and regulators of DNA methylation. Therefore, It seems that MM3 recruits $\mathrm{H} 3 \mathrm{~K} 9 \mathrm{me} 2$ demethylases and thereby eliminates the indispensability of additional inhibition of H3K9me2. Possibly, UNC0638 demethylases the M3 in MM3 and thereby hinders the enhancement of reprogramming by this fusion protein. However, the exact mechanisms of this theory are not clear. It seems correct that for efficient cardiac reprogramming, the suppressive modifications should be inhibited at the early phase of reprogramming. Dzau and colleagues reported that the miRNA combo downregulated expression of the histone lysine N-methyltransferase Setdb2 which specifically trimethylates H3K9. This indicated that the miR combo induced reprogramming by alleviating the suppression of cardiac genes in fibroblasts [115]. In another study, Qian and colleagues reported that during cardiac reprogramming mediated by polycistronic MGT (the most optimum condition) [47], H3K27me3 reduced and $\mathrm{H} 3 \mathrm{~K} 4 \mathrm{me} 3$ increased at cardiac promoters as early as day 3, which was paralleled by a significant, rapid increase in their mRNA expressions [107]. H3K27me3 at loci encoding fibroblast marker genes did not increase until day 10 and $\mathrm{H} 3 \mathrm{~K} 4 \mathrm{me} 3$ progressively decreased along with the reprogramming process. These changes were accompanied by a gradual decrease in mRNA expression of fibroblast marker genes. Overall, cardiac loci lost H3K27me3 and gained $\mathrm{H} 3 \mathrm{~K} 4 \mathrm{me} 3$ as early as day 3 during $\mathrm{iCM}$ generation; fibroblast loci gradually lost H3K4me3 and eventually gained H3K27me3 during this process. In addition to histone modifications, DNA demethylation at specific CpGs of cardiac promotors occurred as early as day 3 [46]. Consistent with other studies, these finding indicated that after early activation of the cardiac program, the fibroblast identity gradually suppressed. 


\section{Mll1 H3K4 methyltransferase activity}

Recently, Liu et al. employed a gain-of-function approach to explore the function of 47 transcription and epigenetic regulators that highly expressed in the cardiac progenitor and neonatal CM stage [58]. The results indicated that Hand2, Mesp1, and Baf60C enhanced cardiac reprogramming mediated by polycistronic MGT, whereas Men1 and Suv39h1 resulted in 5- and 3-fold reductions in cardiac reprogramming as measured by cardiac gene expression and the number of $\alpha \mathrm{MHC}-\mathrm{GFP}+$ cells. These results have shown that Men1 and Suv39h1 are barriers to iCM reprogramming. Men1 is an essential component of a MLL/SET1 histone methyltransferase (HMT) complex that specifically methylates Lys-4 of histone H3 (H3K4). Suv39h1 is an HMT that specifically trimethylates Lys-9 of histone H3 using monomethylated H3 Lys-9 as a substrate. They have observed that inhibition of Suv39h1 by chaetocin did not improve reprogramming efficiency, whereas inhibition of Men1 by MI503 increased reprogramming 1.5fold. It is known that MI503 targets Men1 by blocking the binding of Men1 to the Mll1 and Mll2 complex. To precisely investigate the role of the Men1/Mll1/Mll2 complex, they have used small molecule MM408 (an inhibitor of Ml11-WDR5 interaction), which specifically inhibits Mll1related H3K4 methyltransferase activity without affecting the activities of other Mll family proteins. The results have indicated that MM408 increased reprogramming efficiency in a dosedependent manner. In addition, Mll1 shRNA knockdown also resulted in an elevated iCM conversion rate, which suggested that inhibition of M111 activities by small molecules MI503 and MM408 increased reprogramming efficiency. They found that MM408 simultaneously upregulated cardiac genes and downregulated fibroblast genes. Interestingly, in the early stage of reprogramming, overexpression of MGT in fibroblasts elevated cardiac gene expression and also 
increased adipocyte gene expression 3 days after transduction. Overall, MM408 directly suppressed the expression of the Mll1 target gene Ebf1, a TF involved in adipocyte differentiation. Adipogenesis markers Fabp4 and Pparg also had decreased expressions after MM408 treatment. On the other hand, knockdown of Ebf1 or Mll1 increased the efficiency of iCM reprogramming, which indicated that the Mll1 inhibitor repressed adipocyte formation mainly through blocking the expression of Ebf1. Although the precise mechanism of MII1 was not clear, it seemed that Mll1 promoted iCM conversion by suppression of adipocyte lineage gene expression [58].

Interestingly, inhibition of Bmil (PcG component) and Mll1 (TrxG component) enhances CM formation. Specifically reduced Bmil expression leads to increased levels of the active histone mark H3K4me3 and reduced levels of repressive H2AK199ub at cardiogenic loci, which lead to expression of the cardiogenic gene. Inhibition of Mll1 activity leads to decreased expression of genes involved in adipocyte formation and consequently, low induction of adipocytes. Therefore, it seems that opposite functions of epigenetic factors may work together to facilitate direct cardiac reprogramming.

\section{Serum}

Fetal bovine serum (FBS) has a number of known and unknown factors, which might induce or inhibit CM generation in vitro. Variations between different batches of serum also exist. It has been demonstrated that the efficiency of CM generation from PSCs is increased in the presence of serum-free media and cardiogenic small molecules and/or growth factors [2]. Initially, Srivastava and colleagues have used a serum based medium (DMEM/M199 plus 10\% FBS) during direct cardiac reprogramming. The following reports also used serum-based media for 
cardiac reprogramming from human and mouse fibroblasts. Today, only a few studies have reported the effects for serum or serum-related factors in direct cardiac reprogramming. In one study, Christoforou et al. screened three different media formulations including two serum based media - a basic fibroblast growth medium (FGM), a CM growth medium (CGM), and a low serum growth medium (LSGM) during cardiac deprogramming mediated by 5F (GTM plus Myocardin and SRF) or 7F (GTM plus Myocardin, SRF, Mesp1 and Baf60C) [51]. They found that FGM and CGM did not induce generation of Actn2+/Tnnt2+ cells, while LSGM readily induced the generation of Actn2+/Tnnt $2+$ cross-striated cells. They showed that JAK inhibitor and VPA significantly increased the efficiency of iCM when cells were cultured in LSGM. Low serum appeared to improve the cytoskeletal maturation of iCMs. In general, low serum conditions significantly decreased the proliferative capacity of starting cells. Cardiac TF caused significant downregulation of cell cycling, hence, it was possible that low serum culture conditions enhanced this effect and thereby improved direct cardiac reprogramming [51]. Ieda and colleagues developed a stepwise protocol for efficient direct reprogramming of mouse fibroblasts to iCMs [52]. They showed that culturing GTM-transduced MEF with serum-based medium (DMEM/M199/FBS) followed by two more weeks with serum-free (FFV) medium that consisted of StemPro-34 plus FGF10, FGF2 and VEGF was the most efficient strategy for direct cardiac reprogramming. They showed that in serum-free medium iCMs began to contract earlier. A greater population of $\alpha \mathrm{MHC}-\mathrm{GFP}+$ cells had spontaneous beating and $\mathrm{Ca}^{2+}$ oscillations compared to FBS, suggesting that FFV increased the generation of functional iCMs. Moreover, addition of FGF10, FGF2, and VEGF to FBS did not increase the reprogramming efficiency, suggesting that the serum might contain inhibitory factors for cardiac reprogramming. Microarray analyses revealed that FFV resulted in higher expression of 1461 genes and lower 
expression of 801 genes compared to gene expression levels observed with FBS treatment. They found that the upregulated genes were related to different cardiac functions, including TF, sarcomeric structures, and ion channels, which suggested that FFV globally activated cardiac programming in mouse fibroblasts [52].

\section{Conclusion and future directions}

In this review, we have comprehensively described the findings and status of research on direct cardiac reprogramming. Unlike pluripotency reprogramming in which Oct4, Sox2, Klf4, and cMyc are the master regulators for reprogramming somatic cells of different species to iPSCs, the master regulators of cardiac reprogramming seem to be species specific (Figure 3). For example, GMT seems to be the master regulator of rodent cardiac reprogramming in vivo and in vitro. It is important to note that other factors such as growth factors and epigenetic regulating factors assist GMT in this regard. It seems that GMT must be included in the final combination for cardiac reprogramming in different species. Other cardiac reprogramming factors such as Hand2, Mesp1, Myocd, miR-1, and miR-133 have the chance to be included in the final combination, especially Myocd which has been reported by all human cardiac reprogramming studies (Table 3). Moreover, in recent years, outstanding findings have been reported which increase our knowledge of cardiac reprogramming with rapid progression in this field. 1) Direct reprogramming is a stepwise event in which many cellular and molecular pathways must be manipulated and controlled. 2) Serum work as inhibitors in direct cardiac reprogramming and serum-free defined media seems to be more reliable for $\mathrm{iCM}$ generation and has been demonstrated for $\mathrm{CM}$ generation from pluripotent stem cells. 3) It seems that working on stoichiometry of cardiac TFs/miRNAs is more useful than seeking new combinations. 
Polycistronic and stoichiometric MTG is becoming the best platform for mouse cardiac reprogramming. It is possible to find a polycistronic/stoichiometric system for direct reprogramming of human fibroblasts. 4) Epigenetic factors (histone modifications, miRNAs, and DNA methylation) strongly control direct cardiac reprogramming. Many identified boosters and barriers exert their roles through these factors. 5) CF is efficiently reprogrammed to cardiac cells than other fibroblasts because it has a more active mark and less suppressive mark in the promotors of cardiac genes, which can easily and rapidly switch these genes on. 6) In vivo direct reprogramming has been improved using a polycistronic/stoichiometric system and small molecules, which increase the hope for cardiac treatment using a direct reprogramming strategy. 7) The GFP reporter system is not a reliable system to investigate the incidence for reprogramming, as well as to determine the efficiency of this process. It has been shown that different combinations cause expression of different genes and generation of different cardiac cells. 


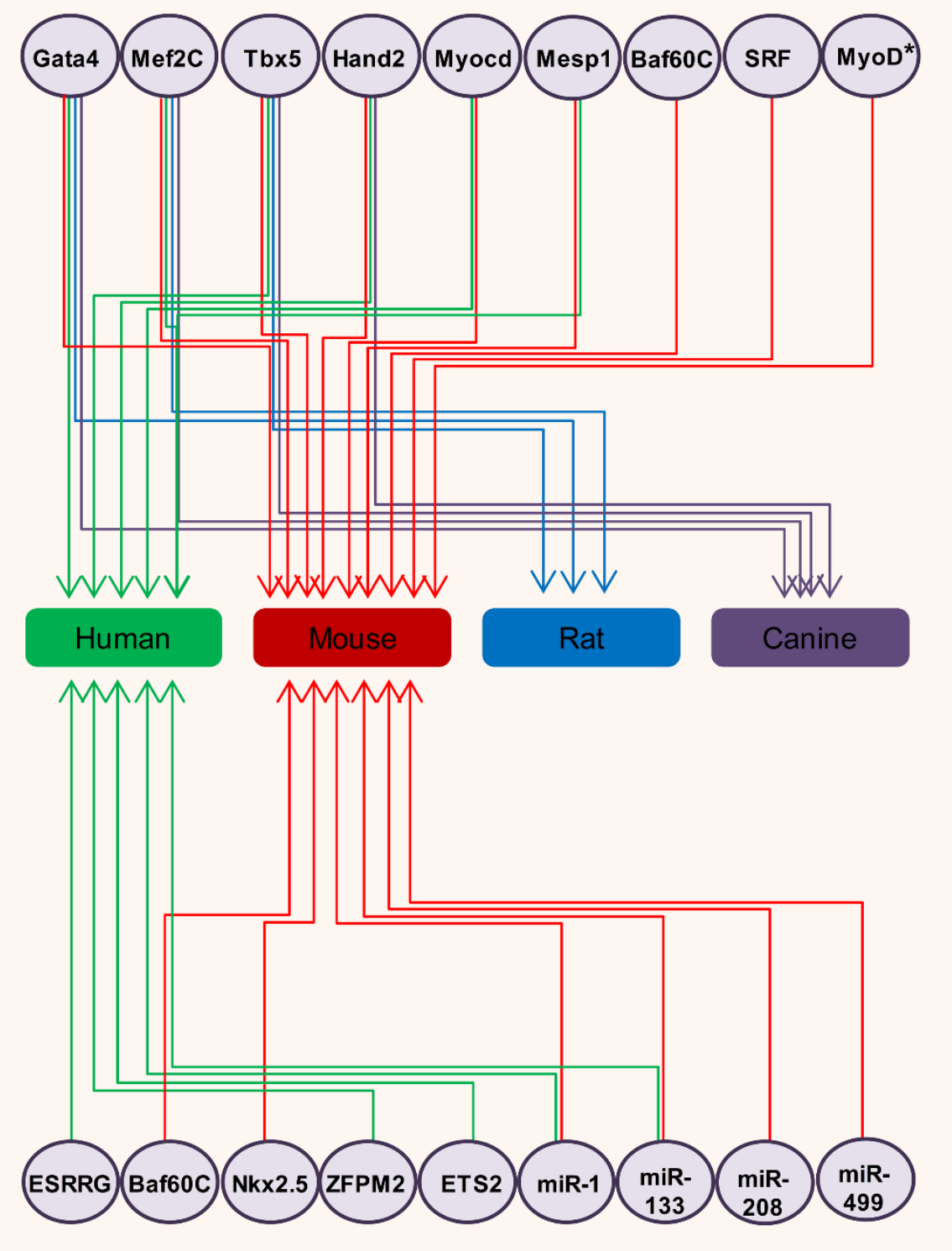

Figure 3. Factors reported for direct cardiac reprogramming in different species. Many transcription factors (TFs) and microRNAs (miRNAs) have been studied for cardiac reprogramming in different species. GTM are necessary for direct cardiac reprogramming in all studied species. Three TFs (Hand2, Myocd, and Mesp1) and two miRNAs; miR-1 and mir-133) are involved in human and mouse direct cardiac reprograming. Esrrg, Zfpm2, and Ets2 are important for humans, while miR-208, miR-499, SRF, Baf60C, and MyoD* have roles in mouse direct cardiac reprogramming. Some factors have inducing roles for one species, which suggests that direct cardiac reprogramming is species specific. (*MyoD: MyoD’ M3 domain which has been fused to Mef2C). 
The efficiency and yield of iCMs is currently very low, however administration of boosters and removal of barriers to cardiac reprogramming will enable researchers to produce a large number of iCMs, which can be utilized in different applications (Figure 4). These applications include: 1) effective cell-based therapy and myocardial repair. iCMs, particularly with the ventricular phenotype, are of great interest for cell-based heart regeneration. 2) High-throughput screening for drug discovery and cardiotoxicity testing. Despite the low efficiency and immature phenotype of iCMs, they have electrophysiologically responded to tested drugs. iCMs can be used to determine SMs that affect CM physiology, metabolism, and survival. 3) Disease modeling. Due to species differences, the data obtained from mice and other animal models are not sufficient and should be complemented by human models. hiCMs can be considered as candidates to model human diseases through the "trial in a dish" models. 5) Cardiac tissue engineering using iCMs can help to create cardiac patches or an entire artificial heart with morphological and functional properties similar to native myocardium. It will be very interesting to investigate cardiac reprogramming of fibroblasts cultured in a 3D culture. This will shed light on the mechanisms of in vivo cardiac reprogramming that occur in the heart. 6) Developmental biology and basic research. iCMs are tremendous tools to decipher the molecular and cellular events that happen for CMs. It is important to note that these cells are not a good choice for studying the dynamic and complex process of cardiogenesis, as their fates are directly switched and many intermediate cardiogenic steps such as early cardiac progenitors' stages are skipped in this process. 


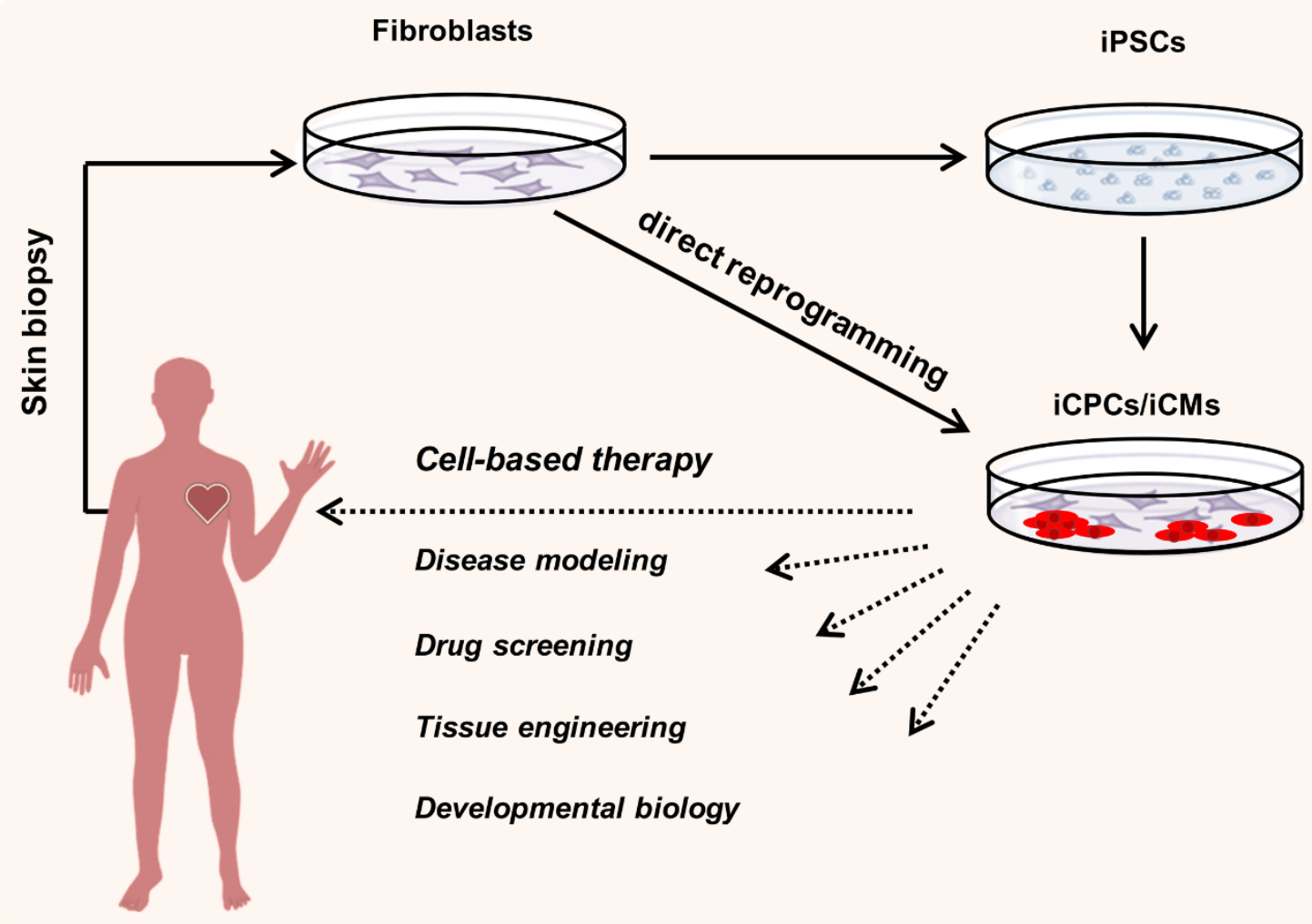

Figure 4. Potential applications of directly reprogrammed cardiac lineage cells (hiCPCs/hiCMs). These cells are generated directly from patient-derived fibroblasts through a safe strategy which skips the pluripotency state. hiCPCs/hiCMs provide a great platform for cardiac cell-based therapy and tissue engineering required functional hiCPCs/hiCMs, especially the ventricular type. hiCPCs/hiCMs, in particular those derived from patient biopsies, are outstanding candidates for new drug discovery, models of cardiac diseases, and enable studies of the developmental processes of the human heart.

Although many aspects of cardiac reprogramming have been studied, numerous research opportunities exist that attempt to answer potential questions in this field. Despite studying different combinations of cardiac TFs/miRNAs and manipulating different cellular and molecular pathways, the efficiency and yield of iCMs remain low. It seems necessary to precisely investigate all mechanisms involved in cardiogenesis in vivo and $\mathrm{CM}$ differentiation in 
vitro. The knowledge obtained from in vitro $\mathrm{CM}$ differentiation which includes cell culture media, coating substrate, inducing and inhibiting factors, oxygen conditions, and cell culture conditions (2D and 3D) will be very useful for direct cardiac reprogramming. The research route that has been following iPSC technology can guide researches to the important aspects of reprogramming potentially involved in cardiac reprogramming. For example, stoichiometry of Oct4, Sox2, Klf4, and C-Myc have already been shown as an important factor for efficient iPSC generation. It is well known that stoichiometry is very important for cardiac reprogramming. Today, it is very clear that cardiogenesis is a stepwise process in which different TFs are expressed at different time points. A hierarchical mechanism appears to be involved in cardiac reprogramming, in which one $\mathrm{TF}$ might work as a pioneer to make the chromatin ready for other TFs. A hierarchical mechanism has been demonstrated for direct reprogramming of fibroblasts to neurons [116].

Although cardiac reprogramming has extensively been investigated in mice, there are few reports of direct reprogramming of human fibroblasts to iCMs. If we consider direct cardiac reprogramming as a strategy for the above mentioned potential applications, it will be necessary to focus on direct cardiac reprogramming of human fibroblasts in greater detail. For example, stoichiometric/polycistronic MGT is becoming the most efficient platform for in vivo and in vitro reprogramming of mouse fibroblasts, while there is no universal and optimized combination for direct cardiac reprogramming in humans. In vivo reprogramming in large animals, especially primates, is an interesting research and the results can pave the way for in vivo cardiac reprogramming in patients. Future studies will determine the reliability of the direct reprogramming strategy for generating CMs required for potential applications. 


\section{Acknowledgements}

This work was supported by grants from Royan Institute, the Iranian Council of Stem Cell

Research and Technology, the Iran National Science Foundation (INSF), and Iran Science Elites

Federation to H.B and Shahid Beheshti University to M.T. 


\section{References}

1. Kikuchi, K. and K.D. Poss, Cardiac regenerative capacity and mechanisms. Annual review of cell and developmental biology, 2012. 28: p. 719-741.

2. Talkhabi, M., N. Aghdami, and H. Baharvand, Human cardiomyocyte generation from pluripotent stem cells: A state-of-art. Life sciences, 2016. 145: p. 98-113.

3. Sanganalmath, S.K. and R. Bolli, Cell therapy for heart failure. Circulation research, 2013. 113(6): p. 810-834.

4. Dixit, P. and R. Katare, Challenges in identifying the best source of stem cells for cardiac regeneration therapy. Stem cell research \& therapy, 2015. 6(1): p. 26.

5. Doppler, S.A., et al., Direct reprogramming-the future of cardiac regeneration? International journal of molecular sciences, 2015. 16(8): p. 17368-17393.

6. Asuelime, G.E. and Y. Shi, A case of cellular alchemy: lineage reprogramming and its potential in regenerative medicine. Journal of molecular cell biology, 2012. 4(4): p. 190-196.

7. Vierbuchen, T. and M. Wernig, Molecular roadblocks for cellular reprogramming. Molecular cell, 2012. 47(6): p. 827-838.

8. Zhao, W., B. Ning, and C. Qian, Regulatory factors of induced pluripotency: current status. Stem Cell Investigation, 2014. 1.

9. Davis, R.L., H. Weintraub, and A.B. Lassar, Expression of a single transfected cDNA converts fibroblasts to myoblasts. Cell, 1987. 51(6): p. 987-1000.

10. Chan, S.S.-K. and M. Kyba, What is a master regulator? Journal of stem cell research \& therapy, 2013. 3.

11. Halder, G., P. Callaerts, and W.J. Gehring, Induction of ectopic eyes by targeting expression of the eyeless gene in Drosophila. Science, 1995. 267(5205): p. 1788-1792.

12. Takahashi, K. and S. Yamanaka, Induction of pluripotent stem cells from mouse embryonic and adult fibroblast cultures by defined factors. cell, 2006. 126(4): p. 663-676.

13. Takahashi, K., et al., Induction of pluripotent stem cells from adult human fibroblasts by defined factors. cell, 2007. 131(5): p. 861-872.

14. Pournasr, B., et al., Concise review: alchemy of biology: generating desired cell types from abundant and accessible cells. Stem cells, 2011. 29(12): p. 1933-1941.

15. Vierbuchen, T. and M. Wernig, Direct lineage conversions: unnatural but useful [quest]. Nature biotechnology, 2011. 29(10): p. 892-907.

16. Zhou, Q., et al., In vivo reprogramming of adult pancreatic exocrine cells to $\beta$-cells. nature, 2008. 455(7213): p. 627-632. 
17. Wei, R. and T. Hong, Lineage reprogramming: a promising road for pancreatic $\beta$ cell regeneration. Trends in Endocrinology \& Metabolism, 2016. 27(3): p. 163-176.

18. Shahbazi, E., et al., Conversion of Human Fibroblasts to Stably Self-Renewing Neural Stem Cells with a Single Zinc-Finger Transcription Factor. Stem cell reports, 2016. 6(4): p. 539551.

19. Pournasr, B., M.H. Asghari-Vostikolaee, and H. Baharvand, Transcription factormediated reprograming of fibroblasts to hepatocyte-like cells. European journal of cell biology, 2015. 94(12): p. 603-610.

20. Batta, K., et al., Direct reprogramming of murine fibroblasts to hematopoietic progenitor cells. Cell reports, 2014. 9(5): p. 1871-1884.

21. Efe, J.A. and S. Ding, Reprogramming, transdifferentiation and the shifting landscape of cellular identity. 2011, Cell Cycle. Taylor \& Francis. p. 1886-1887.

22. Ma, T., et al., Progress in the reprogramming of somatic cells. Circulation research, 2013. 112(3): p. 562-574.

23. Talkhabi, M., et al., Ascorbic acid promotes the direct conversion of mouse fibroblasts into beating cardiomyocytes. Biochemical and biophysical research communications, 2015. 463(4): p. 699-705.

24. Szabo, E., et al., Direct conversion of human fibroblasts to multilineage blood progenitors. Nature, 2010. 468(7323): p. 521-526.

25. Efe, J.A., et al., Conversion of mouse fibroblasts into cardiomyocytes using a direct reprogramming strategy. Nature cell biology, 2011. 13(3): p. 215-222.

26. Garg, V., et al., GATA4 mutations cause human congenital heart defects and reveal an interaction with TBX5. Nature, 2003. 424(6947): p. 443-447.

27. Qian, L. and D. Srivastava, Direct cardiac reprogramming. Circulation research, 2013. 113(7): p. 915-921.

28. Takeuchi, J.K. and B.G. Bruneau, Directed transdifferentiation of mouse mesoderm to heart tissue by defined factors. Nature, 2009. 459(7247): p. 708-711.

29. Ieda, M., et al., Direct reprogramming of fibroblasts into functional cardiomyocytes by defined factors. Cell, 2010. 142(3): p. 375-386.

30. Ieda, M., et al., Cardiac fibroblasts regulate myocardial proliferation through $\beta 1$ integrin signaling. Developmental cell, 2009. 16(2): p. 233-244.

31. David, R., et al., MesP1 drives vertebrate cardiovascular differentiation through Dkk-1mediated blockade of Wnt-signalling. Nature cell biology, 2008. 10(3): p. 338-345. 
32. Song, K., et al., Heart repair by reprogramming non-myocytes with cardiac transcription factors. Nature, 2012. 485(7400): p. 599-604.

33. Ifkovits, J.L., et al., Inhibition of TGF $\beta$ signaling increases direct conversion of fibroblasts to induced cardiomyocytes. PloS one, 2014. 9(2): p. e89678.

34. Hirai, H., et al., Accelerated direct reprogramming of fibroblasts into cardiomyocyte-like cells with the MyoD transactivation domain. Cardiovascular research, 2013: p. cvt167.

35. Protze, S., et al., A new approach to transcription factor screening for reprogramming of fibroblasts to cardiomyocyte-like cells. Journal of molecular and cellular cardiology, 2012. 53(3): p. 323-332.

36. Zhou, L., et al., Cardiac gene activation analysis in mammalian non-myoblasic cells by Nkx2-5, Tbx5, Gata4 and Myocd. PLoS One, 2012. 7(10): p. e48028.

37. Zhou, H., et al., Aktl/protein kinase B enhances transcriptional reprogramming of fibroblasts to functional cardiomyocytes. Proceedings of the National Academy of Sciences, 2015. 112(38): p. 11864-11869.

38. Hirai, H. and N. Kikyo, Inhibitors of suppressive histone modification promote direct reprogramming of fibroblasts to cardiomyocyte-like cells. Cardiovascular research, 2014: p. cvu023.

39. Zhou, Y., et al., Bmil is a key epigenetic barrier to direct cardiac reprogramming. Cell Stem Cell, 2016. 18(3): p. 382-395.

40. Sia, J., et al., Effect of biophysical cues on reprogramming to cardiomyocytes. Biomaterials, 2016. 103: p. 1-11.

41. Vaseghi, H.R., et al., Generation of an inducible fibroblast cell line for studying direct cardiac reprogramming. genesis, 2016. 54(7): p. 398-406.

42. Li, Q., et al., Gata4, Tbx5 and Baf60c induce differentiation of adipose tissue-derived mesenchymal stem cells into beating cardiomyocytes. The international journal of biochemistry \& cell biology, 2015. 66: p. 30-36.

43. Zhao, Y., et al., High-efficiency reprogramming of fibroblasts into cardiomyocytes requires suppression of pro-fibrotic signalling. Nature communications, 2015. 6.

44. Nam, Y.-J., et al., Induction of diverse cardiac cell types by reprogramming fibroblasts with cardiac transcription factors. Development, 2014. 141(22): p. 4267-4278.

45. Qian, L., et al., In vivo reprogramming of murine cardiac fibroblasts into induced cardiomyocytes. Nature, 2012. 485(7400): p. 593-598. 
46. Wang, Y., et al., Hypoxia enhances direct reprogramming of mouse fibroblasts to cardiomyocyte-like cells. Cellular Reprogramming (Formerly" Cloning and Stem Cells"), 2016. 18(1): p. 1-7.

47. Wang, L., et al., Stoichiometry of Gata4, Mef2c, and Tbx5 Influences the Efficiency and Quality of Induced Cardiac Myocyte ReprogrammingNovelty and Significance. Circulation research, 2015. 116(2): p. 237-244.

48. Chen, J.X., et al., Inefficient reprogramming of fibroblasts into cardiomyocytes using Gata4, Mef2c, and Tbx5. Circulation research, 2012: p. CIRCRESAHA. 112.270264.

49. Inagawa, K., et al., Induction of Cardiomyocyte-Like Cells in Infarct Hearts by Gene Transfer of Gata4, Mef2c, and Tbx5Novelty and Significance. Circulation research, 2012. 111(9): p. 1147-1156.

50. Ma, H., et al., In vivo cardiac reprogramming using an optimal single polycistronic construct. Cardiovascular research, 2015. 108(2): p. 217-219.

51. Christoforou, N., et al., Transcription factors MYOCD, SRF, Mesp1 and SMARCD3 enhance the cardio-inducing effect of GATA4, TBX5, and MEF2C during direct cellular reprogramming. PloS one, 2013. 8(5): p. e63577.

52. Yamakawa, H., et al., Fibroblast growth factors and vascular endothelial growth factor promote cardiac reprogramming under defined conditions. Stem cell reports, 2015. 5(6): p. 1128-1142.

53. Jayawardena, T.M., et al., MicroRNA-Mediated In Vitro and In Vivo Direct Reprogramming of Cardiac Fibroblasts to CardiomyocytesNovelty and Significance. Circulation research, 2012. 110(11): p. 1465-1473.

54. Jayawardena, T.M., et al., MicroRNA induced cardiac reprogramming in vivo: evidence for mature cardiac myocytes and improved cardiac function. Circulation research, 2014: $\mathrm{p}$. CIRCRESAHA. 114.304510.

55. Muraoka, N., et al., MiR- 133 promotes cardiac reprogramming by directly repressing Snail and silencing fibroblast signatures. The EMBO journal, 2014. 33(14): p. 1565-1581.

56. Lee, K., et al., Peptide-enhanced mRNA transfection in cultured mouse cardiac fibroblasts and direct reprogramming towards cardiomyocyte-like cells. International journal of nanomedicine, 2015. 10: p. 1841-1854.

57. Wang, X., et al., Selenium Augments microRNA Directed Reprogramming of Fibroblasts to Cardiomyocytes via Nanog. Scientific reports, 2016. 6;23017.

58. Liu, L., et al., Targeting Mll1 H3K4 methyltransferase activity to guide cardiac lineage specific reprogramming of fibroblasts. Cell Discovery, 2016. 2: p. 16036. 
59. Mohamed, T.M., et al., Chemical Enhancement of In Vitro and In Vivo Direct Cardiac Reprogramming. Circulation, 2016: p. CIRCULATIONAHA. 116.024692.

60. Fu, J.-D., et al., Direct reprogramming of human fibroblasts toward a cardiomyocyte-like state. Stem cell reports, 2013. 1(3): p. 235-247.

61. Wada, R., et al., Induction of human cardiomyocyte-like cells from fibroblasts by defined factors. Proceedings of the National Academy of Sciences, 2013. 110(31): p. 12667-12672.

62. Nam, Y.-J., et al., Reprogramming of human fibroblasts toward a cardiac fate. Proceedings of the National Academy of Sciences, 2013. 110(14): p. 5588-5593.

63. Rajarajan, K., M.C. Engels, and S.M. Wu, Reprogramming of mouse, rat, pig, and human fibroblasts into iPS cells. Current protocols in molecular biology, 2012: p. 23.15. 1-23.15. 32.

64. Victor, M.B., et al., Generation of human striatal neurons by microRNA-dependent direct conversion of fibroblasts. Neuron, 2014. 84(2): p. 311-323.

65. Ghosh, J.C., et al., Adaptive mitochondrial reprogramming and resistance to PI3K therapy. Journal of the National Cancer Institute, 2015. 107(3): p. dju502.

66. Islas, J.F., et al., Transcription factors ETS2 and MESP1 transdifferentiate human dermal fibroblasts into cardiac progenitors. Proceedings of the National Academy of Sciences, 2012. 109(32): p. 13016-13021.

67. Pratico, E.D., et al., RNA-mediated reprogramming of primary adult human dermal fibroblasts into c-Kit+ cardiac progenitor cells. Stem cells and development, 2015. 24(22): p. 2622-2633.

68. Ivey, M.J. and M.D. Tallquist, Defining the Cardiac Fibroblast. Circulation Journal, 2016. 80(11): p. 2269-2276.

69. Furtado, M.B., M.W. Costa, and N.A. Rosenthal, The cardiac fibroblast: Origin, identity and role in homeostasis and disease. Differentiation, 2016. 92(3): p. 93-101.

70. Ali, S.R., et al., Developmental Heterogeneity of Cardiac Fibroblasts Does Not Predict Pathological Proliferation and ActivationNovelty and Significance. Circulation research, 2014. 115(7): p. 625-635.

71. Kanisicak, O., et al., Genetic lineage tracing defines myofibroblast origin and function in the injured heart. Nature Communications, 2016. 7.

72. Mathison, M., et al., In vivo cardiac cellular reprogramming efficacy is enhanced by angiogenic preconditioning of the infarcted myocardium with vascular endothelial growth factor. Journal of the American Heart Association, 2012 . 1(6): p. e005652.

73. Palazzolo, G., et al., Cardiac niche influences the direct reprogramming of canine fibroblasts into cardiomyocyte-like cells. Stem cells international, 2015. 2016. 
74. $\mathrm{Hu}, \mathrm{Y} . \mathrm{F}$., et al., Biological pacemaker created by minimally invasive somatic reprogramming in pigs with complete heart block. Science translational medicine, 2014. 6(245): p. 245ra94-245ra94.

75. Kapoor, N., et al., Direct conversion of quiescent cardiomyocytes to pacemaker cells by expression of Tbx18. Nature biotechnology, 2013. 31(1): p. 54-62.

76. Mathison, M., et al., "Triplet" polycistronic vectors encoding Gata4, Mef2c, and Tbx5 enhances postinfarct ventricular functional improvement compared with singlet vectors. The Journal of thoracic and cardiovascular surgery, 2014. 148(4): p. 1656-1664. e2.

77. Bruneau, B.G., Signaling and transcriptional networks in heart development and regeneration. Cold Spring Harbor perspectives in biology, 2013. 5(3): p. a008292.

78. Liu, W. and A.C. Foley, Signaling pathways in early cardiac development. Wiley Interdisciplinary Reviews: Systems Biology and Medicine, 2011. 3(2): p. 191-205.

79. Jopling, C., et al., Hypoxia induces myocardial regeneration in zebrafish. Circulation, 2012: p. CIRCULATIONAHA. 112.107888.

80. J Patterson, A. and L. Zhang, Hypoxia and fetal heart development. Current molecular medicine, 2010. 10(7): p. 653-666.

81. Dunwoodie, S.L., The role of hypoxia in development of the Mammalian embryo. Developmental cell, 2009. 17(6): p. 755-773.

82. Correia, C., et al., Combining hypoxia and bioreactor hydrodynamics boosts induced pluripotent stem cell differentiation towards cardiomyocytes. Stem Cell Reviews and Reports, 2014. 10(6): p. 786-801.

83. Nakada, Y., et al., Hypoxia induces heart regeneration in adult mice. Nature, 2017. 541(7636): p. 222-227.

84. Prickaerts, P., et al., Hypoxia increases genome-wide bivalent epigenetic marking by specific gain of H3K27me3. Epigenetics \& Chromatin, 2016. 9(1): p. 46.

85. Park, J., et al., Control of stem cell fate and function by engineering physical microenvironments. Integrative Biology, 2012. 4(9): p. 1008-1018.

86. Downing, T.L., et al., Biophysical regulation of epigenetic state and cell reprogramming. Nature materials, 2013. 12(12): p. 1154-1162.

87. Zhu, R., et al., Physical developmental cues for the maturation of human pluripotent stem cell-derived cardiomyocytes. Stem cell research \& therapy, 2014. 5(5): p. 117.

88. Lundy, S.D., et al., Structural and functional maturation of cardiomyocytes derived from human pluripotent stem cells. Stem cells and development, 2013. 22(14): p. 1991-2002. 
89. Morez, C., et al., Enhanced efficiency of genetic programming toward cardiomyocyte creation through topographical cues. Biomaterials, 2015. 70: p. 94-104.

90. Xavier-Neto, J., et al., Signaling through retinoic acid receptors in cardiac development: doing the right things at the right times. Biochimica et Biophysica Acta (BBA)-Gene Regulatory Mechanisms, 2015. 1849(2): p. 94-111.

91. van Weerd, J.H., et al., Epigenetic factors and cardiac development. Cardiovascular research, 2011. 91(2): p. 203-211.

92. Carey, B.W., et al., Reprogramming factor stoichiometry influences the epigenetic state and biological properties of induced pluripotent stem cells. Cell stem cell, 2011. 9(6): p. 588598.

93. Itoh, N., et al., Roles of FGF signals in heart development, health, and disease. Frontiers in Cell and Developmental Biology, 2016. 4.

94. Kitamura, Y., et al., Manipulation of cardiac phosphatidylinositol 3-kinase (PI3K)/Akt signaling by apoptosis regulator through modulating IAP expression (ARIA) regulates cardiomyocyte death during doxorubicin-induced cardiomyopathy. Journal of Biological Chemistry, 2014. 289(5): p. 2788-2800.

95. Lien, E.C., C.A. Lyssiotis, and L.C. Cantley, Metabolic Reprogramming by the PI3KAkt-mTOR Pathway in Cancer, in Metabolism in Cancer. 2016, Springer. p. 39-72.

96. Kivelä, R., et al., Vegf-B-induced vascular growth leads to metabolic reprogramming and ischemia resistance in the heart. EMBO molecular medicine, 2014. 6(3): p. 307-321.

97. Beis, D., S. Kalogirou, and N. Tsigkas, Insights into Heart Development and Regeneration, in Introduction to Translational Cardiovascular Research. 2015, Springer. p. 1730.

98. Bianco, C., et al., Cripto-1 is required for hypoxia to induce cardiac differentiation of mouse embryonic stem cells. The American journal of pathology, 2009. 175(5): p. 2146-2158.

99. Li, X.-H., et al., Generation of Functional Human Cardiac Progenitor Cells by HighEfficiency Protein Transduction. Stem cells translational medicine, 2015. 4(12): p. 1415-1424.

100. Ozhan, G. and G. Weidinger, Wnt/ $\beta$-catenin signaling in heart regeneration. Cell Regeneration, 2015. 4(1): p. 3.

101. Mohri, T., et al., JAK-STAT signaling in cardiomyogenesis of cardiac stem cells. Jak-stat, 2012. 1(2): p. 125-130.

102. Lalit, P.A., et al., Lineage reprogramming of fibroblasts into proliferative induced cardiac progenitor cells by defined factors. Cell Stem Cell, 2016. 18(3): p. 354-367. 
103. Nakashima, Y. and T. Omasa, What Kind of Signaling Maintains Pluripotency and Viability in Human-Induced Pluripotent Stem Cells Cultured on Laminin-511 with Serum-Free Medium? BioResearch open access, 2016. 5(1): p. 84-93.

104. Mohsin, S., et al., Empowering adult stem cells for myocardial regeneration. Circulation research, 2011. 109(12): p. 1415-1428.

105. Bujak, M. and N.G. Frangogiannis, The role of TGF- $\beta$ signaling in myocardial infarction and cardiac remodeling. Cardiovascular research, 2007. 74(2): p. 184-195.

106. Goumans, M.-J., et al., TGF- $\beta 1$ induces efficient differentiation of human cardiomyocyte progenitor cells into functional cardiomyocytes in vitro. Stem cell research, 2008. 1(2): p. 138149.

107. Liu, Z., et al., Re-patterning of H3K27me3, H3K4me3 and DNA methylation during fibroblast conversion into induced cardiomyocytes. Stem cell research, 2016. 16(2): p. 507-518.

108. Fang, F., et al., Early growth response 3 (Egr-3) is induced by transforming growth factor- $\beta$ and regulates fibrogenic responses. The American journal of pathology, 2013. 183(4): p. 1197-1208.

109. Sauvageau, M. and G. Sauvageau, Polycomb group proteins: multi-faceted regulators of somatic stem cells and cancer. Cell stem cell, 2010. 7(3): p. 299-313.

110. Boros, J., et al., Polycomb repressive complex 2 and H3K27me3 cooperate with H3K9 methylation to maintain heterochromatin protein $1 \alpha$ at chromatin. Molecular and cellular biology, 2014. 34(19): p. 3662-3674.

111. Wang, Q.T., Epigenetic regulation of cardiac development and function by polycomb group and trithorax group proteins. Developmental Dynamics, 2012. 241(6): p. 1021-1033.

112. Wamstad, J.A., et al., Dynamic and coordinated epigenetic regulation of developmental transitions in the cardiac lineage. Cell, 2012. 151(1): p. 206-220.

113. Valiente-Alandi, I., et al., Bmil + cardiac progenitor cells contribute to myocardial repair following acute injury. Stem Cell Research \& Therapy, 2016. 7(1): p. 100.

114. Hirai, H., et al., Efficient iPS cell production with the MyoD transactivation domain in serum-free culture. PloS one, 2012. 7(3): p. e34149.

115. Hodgkinson, C.P., et al., MicroRNAs and cardiac regeneration. Circulation research, 2015. 116(10): p. 1700-1711.

116. Wapinski, O.L., et al., Hierarchical mechanisms for direct reprogramming of fibroblasts to neurons. Cell, 2013. 155(3): p. 621-635. 


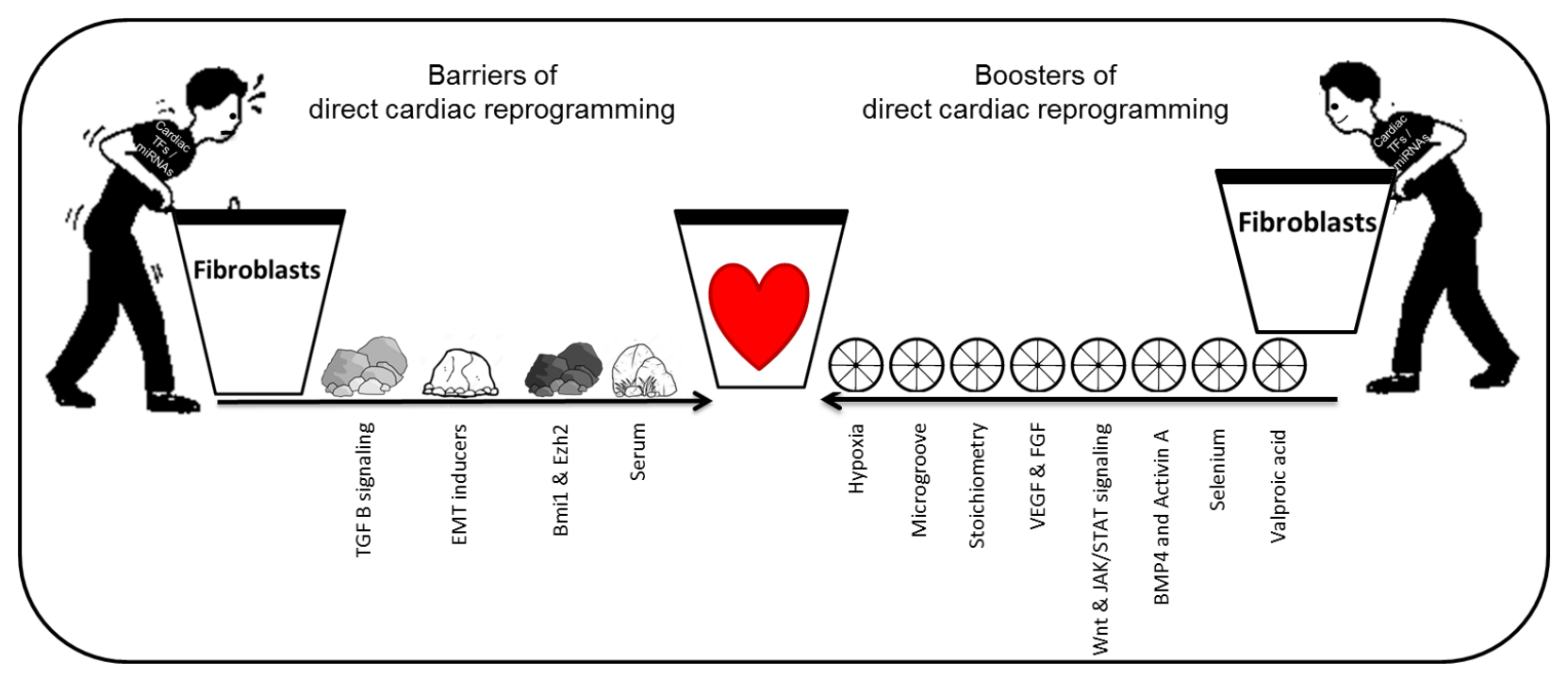

Graphical abstract 